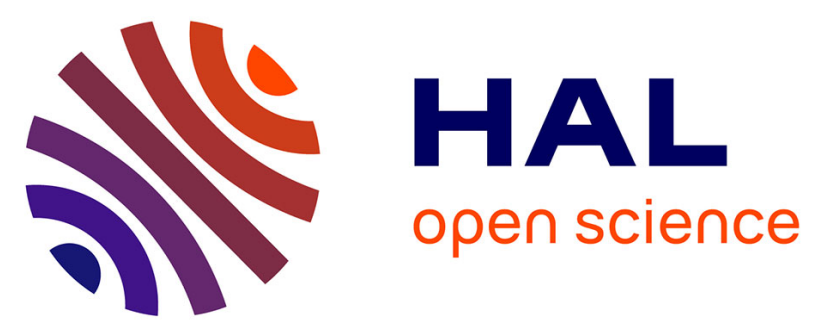

\title{
BCL-XL blockage in TNBC models confers vulnerability to inhibition of specific cell cycle regulators
}

Olivier Castellanet, Fahmida Ahmad, Yaron Vinik, Gordon Mills, Bianca Habermann, Jean-Paul Borg, Sima Lev, Fabienne Lamballe, Flavio Maina

\section{- To cite this version:}

Olivier Castellanet, Fahmida Ahmad, Yaron Vinik, Gordon Mills, Bianca Habermann, et al.. BCLXL blockage in TNBC models confers vulnerability to inhibition of specific cell cycle regulators. Theranostics, 2021, 11 (19), pp.9180-9197. 10.7150/thno.60503 . hal-03429754

\section{HAL Id: hal-03429754 https://hal.science/hal-03429754}

Submitted on 20 Nov 2021

HAL is a multi-disciplinary open access archive for the deposit and dissemination of scientific research documents, whether they are published or not. The documents may come from teaching and research institutions in France or abroad, or from public or private research centers.
L'archive ouverte pluridisciplinaire HAL, est destinée au dépôt et à la diffusion de documents scientifiques de niveau recherche, publiés ou non, émanant des établissements d'enseignement et de recherche français ou étrangers, des laboratoires publics ou privés. 
Research Paper

\title{
BCL-XL blockage in TNBC models confers vulnerability to inhibition of specific cell cycle regulators
}

\author{
Olivier Castellanet ${ }^{1 *}$, Fahmida Ahmad ${ }^{1 *}$, Yaron Vinik ${ }^{2}$, Gordon B. Mills ${ }^{3}$, Bianca Habermann ${ }^{1}$, Jean-Paul \\ Borg $^{4,5}$, Sima Lev², Fabienne Lamballe ${ }^{1 凶}$, Flavio Maina ${ }^{\circledR}$ \\ 1. Aix Marseille Univ, CNRS, Developmental Biology Institute of Marseille (IBDM), Turing Center for Living Systems, Parc Scientifique de Luminy, Marseille \\ (France). \\ 2. Weizmann Institute of Science, Department of Molecular Cell Biology, Rehovot (Israel). \\ 3. Knight Cancer Institute, Portland, OR 97201, USA. \\ 4. Aix Marseille Univ, Centre de Recherche en Cancérologie de Marseille (CRCM), Equipe labellisée Ligue 'Cell polarity, cell signaling and cancer', Inserm, \\ CNRS, Institut Paoli-Calmettes, Marseille (France). \\ 5. Institut Universitaire de France (IUF) \\ ${ }^{*}$ Co-first authors. \\ $\triangle$ Corresponding authors: Flavio Maina \& Fabienne Lamballe shared senior authorship. fabienne.lamballe@univ-amu.fr; flavio.maina@univ-amu.fr.
}

(C) The author(s). This is an open access article distributed under the terms of the Creative Commons Attribution License (https://creativecommons.org/licenses/by/4.0/). See http://ivyspring.com/terms for full terms and conditions.

Received: 2021.03.16; Accepted: 2021.08.23; Published: 2021.09.03

\begin{abstract}
Cell cycle regulators are frequently altered in Triple-Negative Breast Cancer (TNBC). Emerging agents targeting these signals offer the possibility to design new combinatorial therapies. However, preclinical models that recapitulate TNBC primary resistance and heterogeneity are essential to evaluate the potency of these combined treatments.

Methods: Bioinformatic processing of human breast cancer datasets was used to analyse correlations between expression levels of cell cycle regulators and patient survival outcome. The MMTV-R26Met mouse model of TNBC resistance and heterogeneity was employed to analyse expression and targeting vulnerability of cell cycle regulators in the presence of BCL-XL blockage. Robustness of outcomes and selectivity was further explored using a panel of human breast cancer cells. Orthotopic studies in nude mice were applied for preclinical evaluation of efficacy and toxicity. Alterations of protein expression, phosphorylation, and/or cellular localisation were analysed by western blots, reverse phase protein array, and immunocytochemistry. Bioinformatics was performed to highlight drug's mechanisms of action.

Results: We report that high expression levels of the $B C L 2 L I$ gene encoding $B C L-X L$ and of specific cell cycle regulators correlate with poor survival outcomes of TNBC patients. Blockage of $B C L-X L$ confers vulnerability to drugs targeting CDK1/2/4, but not FOXM1, CDK4/6, Aurora $A$ and Aurora $B$, to all MMTV-R26Met and human TNBC cell lines tested. Combined blockage of BCL-XL and CDK1/2/4 interfered with tumour growth in vivo. Mechanistically, we show that, co-targeting of BCL-XL and CDK1/2/4 synergistically inhibited cell viability by combinatorial depletion of survival and RTK/AKT signals, and concomitantly restoring FOXO3a tumour suppression actions. This was accompanied by an accumulation of DNA damage and consequently apoptosis.

Conclusions: Our studies illustrate the possibility to exploit the vulnerability of TNBC cells to CDK1/2/4 inhibition by targeting BCL-XL. Moreover, they underline that specificity matters in targeting cell cycle regulators for combinatorial anticancer therapies.
\end{abstract}

Key words: cancer mouse model, triple-negative breast cancer, cell cycle regulators, BCL-XL, MET

\section{Introduction}

Targeting of the anti-apoptotic protein BCL-XL together with anti-mitotic agents has been proposed as an efficient therapeutic strategy for different human cancers, including triple-negative breast cancer (TNBC), a particular aggressive subtype of breast cancer [1]. TNBC is defined by the lack of 
oestrogen (ER) and progesterone (PR) receptors and by the absence of amplification/overexpression of the HER2 receptor tyrosine kinase (RTK) [2]. The disease accounts for $\sim 15 \%$ of all breast cancer types and is characterised by an extraordinary molecular heterogeneity. Current therapeutic options for targeted treatment are limited due to primary or acquired resistance, and major efforts are devoted to search for molecular alterations and predict vulnerabilities for effective targeted therapy [3]. However, the high heterogeneity of TNBC challenges the identification of generic targets and highlights the requirement for precision therapy and preclinical models that recapitulate TNBC heterogeneity.

We have recently reported the generation of a rather unique mouse model in which a subtle increase in the wild-type MET RTK expression levels in the mammary gland (MMTV-R26 $6^{\text {Met }}$ mice) leads to spontaneous tumour formation faithfully recapitulating TNBC features [4]. These include histological, molecular, and signalling heterogeneity, as well as primary resistance to chemotherapy and approved targeted therapies [5, 6]. We further exploited the $M M T V-R 26^{\text {Met }}$ TNBC model to identify a highly effective therapeutic protocol based on the combined inhibition of the anti-apoptotic molecule BCL-XL and of the cell cycle checkpoint regulator WEE1 [4]. Cell cycle proteins are frequently overexpressed and/or overactivated in several cancer types including TNBC. For example, loss of RB or p16 $16^{\mathrm{INK} 4}$ is frequent across TNBC subtypes $[7,8]$. Alterations in cyclin D and E, CDK4/6, and CDK2 in TNBC have also been reported [9]. Furthermore, mutations in p53, a master cell cycle regulator of the G1/S checkpoint, are present in a large proportion of TNBC, thus leaving cells to mainly rely on the G2/M checkpoint to maintain DNA integrity [10, 11]. Therefore, targeting cell cycle regulators provide a clear rationale for designing anti-cancer therapy [10]. The vulnerability of TNBC to inhibitors of cell cycle regulators has been demonstrated by several studies using different drugs targeting distinct signalling components. For example, the CDK4/ 6 inhibitor Palbociclib, effectively used in ER-positive breast cancer, has recently been explored in combination with chemotherapy in RB-positive TNBC cell models [12-14]. Additionally, it has been reported that a combinatorial treatment based on CDK4/ 6 plus BET inhibitors leads to cell division errors and death, although leading to the emergence of heterogeneous mechanisms of resistance [15].

In the present study, we employed the MMTV-R26 $6^{\text {Met }}$ model system that recapitulates TNBC heterogeneity and primary resistance to explore the effect of inhibiting specific cell cycle regulators while blocking BCL-XL function. Our results show that targeting specific cell cycle components is important and uncover a detrimental effect on TNBC cells of CDK1/2/4 plus BCL-XL inhibition in vitro and in vivo. We provide evidence that this combinatorial targeting significantly reduces the levels of RTK and AKT signalling besides perturbing cell cycle and DNA repair.

\section{Methods}

\section{Mouse cell lines}

$M M T V-R 26^{\text {Met }}$ MGT cell lines were derived from independent $M M T V-R 26^{M e t}$ tumours and established as previously described [4]. The establishment of normal mammary epithelial cell cultures was also described in [4]. Cells were cultured in complete DMEM/F12 (Dulbecco's modified Eagle's media/F12, $1 / 1$, ThermoFisher Scientific) medium (DMEM/F12, supplemented with $10 \%$ foetal bovine serum (FBS, ThermoFisher Scientific), penicillin-streptomycin $(\mathrm{P} / \mathrm{S}, 100 \mathrm{U} / \mathrm{mL} / 0.1 \mathrm{mg} / \mathrm{mL}$, ThermoFisher Scientific), glutamine (2 $\mathrm{mM}$, ThermoFisher Scientific), glucose $(0.25 \%$, Sigma), insulin $(10 \mu \mathrm{g} / \mathrm{mL}$, Sigma), transferrin $(10 \mu \mathrm{g} / \mathrm{mL}$, Sigma), sodium selenite (5 $\mathrm{ng} / \mathrm{mL}$, Sigma), hydrocortisone $(0.5 \mu \mathrm{g} / \mathrm{mL}$, Sigma), EGF (20 ng/mL, Roche), and HGF (10 ng/mL, Peprotech), at $37{ }^{\circ} \mathrm{C}$ in a $5 \% \mathrm{CO}_{2}$ atmosphere. PCR-based assays were performed on all cell lines to verify that they were free of Mycoplasma contamination. Mouse embryonic fibroblasts were kindly provided by P. Perrin, either untreated or treated with Mitomycin C (Sigma).

\section{Human cell lines}

All human TNBC (MDA-MB-231, MDA-MB-468, SUM-159, Hs578t, HCC-1937, and BT-549) and non-TNBC (MCF-7, SKBR-3, and BT-474) cell lines used in this study were obtained from the American Type Culture Collection (ATCC) without further authentication and were tested by PCR-based assay to verify that they were free of Mycoplasma contamination. These human breast cancer cells were grown in DMEM/F12 medium supplemented with $\mathrm{P} / \mathrm{S}$, glutamine $(2 \mathrm{mM})$, sodium pyruvate $(1 \mathrm{mM}$, ThermoFisher Scientific), non-essential amino acids (ThermoFisher Scientific), and insulin $(10 \mu \mathrm{g} / \mathrm{mL})$.

\section{Animals}

All procedures involving the use of animals were carried out in accordance with the European Community Council Directive of 22 September 2010 on the protection of animals used for experimental purposes (2010/63/EU). The experimental protocols were performed according to the institutional Ethical Committee guidelines for animal research (Comité 
d'éthique pour l'expérimentation animale - Comité d'éthique de Marseille) and in compliance with the French law under the agreement number D13-055-21, delivered by the "Préfecture de la Région ProvenceAlpes-Côte-d'Azur et des Bouches-du-Rhône". The mouse project authorization of the Maina laboratory is: APAFIS \#8214-2016121417291352.v5, delivered by the "Ministère de l'Enseignement Supérieur, de la Recherche et de l'Innovation". For orthotopic studies, MDA-MB-231 cells $\left(2 \times 10^{6}\right)$ were injected bilaterally into the fourth mammary fat pad of 6-week-old female nude (Rj:NMRI-Foxn1 nu/nu) mice. Eleven days later, mice were randomized into two groups (n $=8$ per group) and treated with either vehicle or the A1155463 + R547 drug combination. A1155463 was administered intraperitoneally (daily, $5 \mathrm{mg} / \mathrm{kg}$ prepared in $2 \%$ DMSO $+30 \%$ PEG300 (Sigma) $+5 \%$ Tween 80). The R547 compound was injected into the tail vein twice per week $(40 \mathrm{mg} / \mathrm{kg}$ prepared in $10 \%$ PEG300 + 90\% hydroxypropyl - $\beta$-cyclodextrin (sol: $28 \%$ in water; Alfa Aesar)). Mice were treated for 20 days. Mouse body weight and tumour progression were monitored 3 times per week. Tumour volumes were determined using a digital calliper and calculated according to the following formula: $\mathrm{V}$ $($ volume $)=\mathrm{L}$ (length) $\times \mathrm{W}(\text { width })^{2} / 2$. At the end of the experiment, mice were sacrificed, tumours were excised. Tumour volumes and weights were then measured.

\section{Drugs}

Drug concentration and sources are reported in Table S1. Calculation of the Synergy maps and Bliss score has been performed with online SynergyFinder tool v1.0 [16] using "Viability" parameter as readout and "Bliss Method" with correction activated. The CompuSyn software v1.0 using the Chou-Talalay equation was applied to measure synergy or additive effects of drug combinations. Combination index $\mathrm{CI}<$ 1 indicates synergism, CI $<0.5$ indicates strong synergism, $\mathrm{CI}=1$ means additive effect and CI $>1$ stands for antagonism.

\section{Cell viability assay}

Cell viability assay was performed on MMTV-R26 $6^{\text {Met }}$ MGT and human breast cancer cells as previously described [4]. Briefly, cells were plated in 96-well plates, and treated after $24 \mathrm{~h}$ with either single or combined drugs at the indicated concentrations. Cell viability was determined $48 \mathrm{~h}$ later using the Cell Titer Glo Luminescent Assay (Promega). Data are mean values of at least three independent experiments done in triplicates.

\section{Cell cycle analysis by flow cytometry}

$M M T V-R 26^{\text {Met }}$ cells were treated for $12 \mathrm{~h}$ with vehicle, A1155463 (A11, $1 \mu \mathrm{M})$, R547 (3 $\mu \mathrm{M})$, alone or in combination. After trypsinisation, cell suspension was then processed as previously described [4]. Three independent experiments were performed.

\section{Immunocytochemistry}

Protocols used were as described in [4]. Percentage of TritonX-100 and antibodies used for immunofluorescence staining are detailed in Table S2. Briefly, for mitotic catastrophe studies, cells treated for $16 \mathrm{~h}$ with the indicated drugs, were immunostained with anti-pH3 and a-Tubulin (microtubules) antibodies. Mitotic catastrophe was analysed in cells in metaphase and anaphase among the pH3-positive cells. In contrast to WEE1 targeting (with Adavosertib), as previously reported in [4], and used here as control, R547 does not induce mitotic catastrophe. In all experiments, DAPI was used to counterstain the nuclear DNA. For FoxO3a localisation studies, quantification of nucleus versus cytoplasmic staining intensity was determined using Image J Intensity Ratio Nuclei Cytoplasm Tool, RRID:SCR_018573.

\section{Western blotting}

Protein extracts were prepared and western blot analysis was performed as previously described [4]. The antibodies used are reported in Table S2. ACTIN and Ponceau staining were used as loading controls. Ponceau stainings are shown in supplementary information (non-edited gels). For densitometric analysis, the intensity of each total protein band was measured using the Image software (National Institutes of Health, USA), and was normalized to its respective ACTIN loading. Quantification of phosphorylated proteins was reported in relation to the total expression of the protein of interest, when relevant (see Table S3).

\section{Reverse phase protein array (RPPA)}

Protein lysates of either MMTV-R26 $6^{\text {Met }}$ tumours and control mammary glands (from MMTV-Cre mice), or MGT cells treated or not with A1155463 (1 $\mu \mathrm{M})$, R547 $(3 \mu \mathrm{M})$, or the drug combination A1155463 $+\operatorname{R} 547(1 \mu \mathrm{M}, 3 \mu \mathrm{M})$ for $12 \mathrm{~h}$, were prepared according to the MD Anderson Cancer Center platform instructions. RPPA of cells treated with Adavosertib $(3 \mu \mathrm{M})$, or A1155463 + Adavosertib (1 $\mu \mathrm{M}, 3 \mu \mathrm{M})$ was previously reported [4], and used in this study to compare signalling changes occurring with the different drug combinations. Samples were screened with 426 antibodies to identify signalling changes in protein expression and phosphorylation levels. 


\section{RNA-seq}

Total RNA from dissected MMTV-R26 $6^{\text {Met }}$ tumours $(\mathrm{n}=4)$ and control mammary gland tissues $(n=3)$ was processed for transcriptome analysis. RNA integrity was assessed using the Agilent RNA 6000 Pico kit and Agilent 2100 Bioanalyzer (Agilent Technologies, Santa Clara, California) according to the manufacturer's instructions. Sequencing was performed as previously described [17].

\section{Quantitative RT-PCR analysis}

Studies were performed as previously described [4]. RNA was extracted from MGT11 cells either untreated or treated with A1155463 + R547 drug combination. Oligos are reported in Table S4. All reactions were run in triplicate and repeated in three independent experiments.

\section{Bioinformatic analysis}

Analysis of publicly available microarray data: Kaplan-Meier curve reporting the probability (in percent) of the overall survival of human TNBC patients according to the BCL2L1 gene encoding BCL-XL, and/or specific cell cycle regulator levels. The NCBI dataset used was the GSE31519 Affymetrix Human Genome U133A Array. The database includes 580 TNBC patients phenotyped by immunohistochemistry.

Raw RNA-seq reads were mapped against the latest release (mm10) of the mouse genome using STAR aligner [18]. We used featureCounts [19] to quantify mapped reads. Differential expression analysis was performed with the RStudio (RStudio, Inc., Boston, MA) software using the DESeq2 package [20]. For data shown in Figure 3C and S3A, a cut-off $P$ value $<0.05$ was applied. The Genseset enrichment analysis (GSEA) software [21, 22] was used on the WikiPathways database to highlight enriched pathways in the MMTV-R26 $6^{\text {Met }}$ tumours versus controls. Ranking of the enriched pathways was then performed using the Enrichr software. RPPA analyses of drug perturbation effects were done using biological duplicates. Expression levels of proteins were $\log 2$ transformed before analysis. GSEA was employed to determine the statistically significant enriched pathways between cells treated with either A1155463 + R547 or A1155463 + Adavosertib. Differential gene expressions were obtained from the RPPA data outcomes. GSEA v4.1.0 was obtained from the Broad Institute (http://software. broadinstitute.org/gsea/index.jsp). The collection of annotated gene sets of REACTOME was obtained from the GSEA website (MSigDB, http://www.gseamsigdb.org/gsea/msigdb/index.jsp).

\section{Statistical analysis}

The probability of overall survival rates was calculated using the Kaplan-Meier method. P values were computed using the Logrank (Mantel Cox) test. $\mathrm{P}$ values are indicated in figures. $\mathrm{P}>0.05$ was considered as non-significant (ns). ${ }^{*} \mathrm{P}<0.05$; ${ }^{* *} \mathrm{P}<$ 0.01 ; ${ }^{* *} \mathrm{P}<0.001$. For RPPA studies, analysis of fold-change proteins and $\mathrm{P}$ values to determine significantly differentially expressed proteins were done by the Limma package in R. Data are presented as the mean \pm standard error of the mean (s.e.m.), according to sample distributions. For two sided comparisons, unpaired Student's $t$ test was used for data showing normal distributions, and Wilcoxon test was used in other situations. For multiple comparisons, we used ANOVA test followed by Tukey test. All statistical analyses were performed using the GraphPad Prism and software.

\section{Results}

\section{High expression levels of the $B C L 2 L I$ gene coding for BCL-XL and of selective cell cycle regulators correlate with poor survival outcomes of TNBC patients}

Recent studies using breast cancer tissue-specific microarray databases (GSE42568, GSE45827, and GSE54002) have reported a strong enrichment in cell cycle pathway genes [23], which we also illustrate in Figure 1A and S1. Common up- and down-regulated genes from these three microarray datasets were highly enriched in cell cycle regulation pathways from KEGG and WikiPathways (Figure 1B, Table S5). Analysis of the Pan-Cancer Atlas cohort of the TCGA database revealed that about $50 \%$ of breast cancer patients have altered cell cycle genes, with breast cancer ranking second among all cancer types analysed for the percentage of amplification events (Figure 1C). Among the four breast cancer sub-types, HER2 $^{+}$and TNBC patients show the highest frequency of alteration in cell cycle regulators (Figure 1D). Gene amplification is the major type of alterations found in breast cancer patients (Figure 1D). We next used a TNBC microarray database (GSE31519; 580 patients) to analyse the relevance of cell cycle gene levels in patient outcomes. We found that TNBC patients with high levels of BCL2L1 or specific cell cycle regulators such as CDK1,CDK6 and WEE1, exhibit a shorter overall survival rate (Figure 2A-B, Table S6). Interestingly, patients who concurrently highly express the anti-apoptotic factor $B C L 2 L 1$ and the cell cycle modulators $C D K 1, C D K 6$, or WEE1, have poor clinical outcome, thus highlighting the detrimental effect of their concomitant high expression levels (Figure 2C, Table S6). Intriguingly, 
high levels of other cell cycle modulators such as CDK2, CDK4, FOXM1, AURKA, or AURKB, either alone or together with $B C L 2 L 1$ levels, is not associated with altered patient survival (Figure 2B,C, Table S6). The apparent significant difference between the survival curves in the combination sets is mainly due to BCL2L1 levels (Figure 2C, Table S6). Furthermore, patients with high levels of CDK1 and WEE1 exhibit more aggressive TNBC tumours (classified as grade 3; Figure 2D). Interestingly, this was also observed taking into consideration co-expression of high versus low BCL2L1 with CDK1 or WEE1 (Figure 2E). This significant increase in aggressiveness was not observed in patients with high levels of $C D K 6$ (Figure $2 \mathrm{D}, \mathrm{E})$. Since BCL-2 and MCL-1 proteins may have overlapping anti-apoptotic functions with BCL-XL in cancer cells, we compared the impact of their mRNA expression levels, either alone or in combination with CDK1, CDK6 and WEE1, on the overall survival of TNBC patients. We found that patients with high levels of $B C L-2$ or $M C L-1$ have a lower survival rate than those with low $B C L-2$ or $M C L-1$ expression levels. Furthermore, TNBC patients with concurrent high levels of either $B C L-2$ or $M C L-1$ and the CDK1, CDK6 or WEE1 cell cycle regulators exhibit poor clinical outcome, as observed for high BCL2L1 expressers (Figure S2A,B). These results suggest a greater implication of specific cell cycle regulators, particularly when overexpressed with BCL2 family members, on survival of TNBC patients.
A

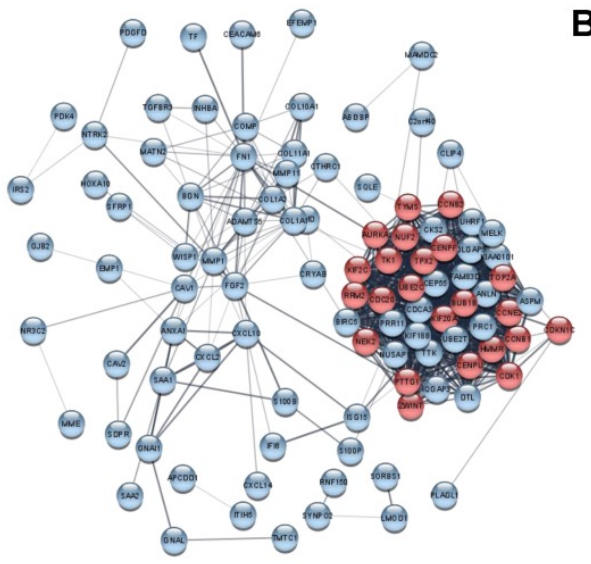

C

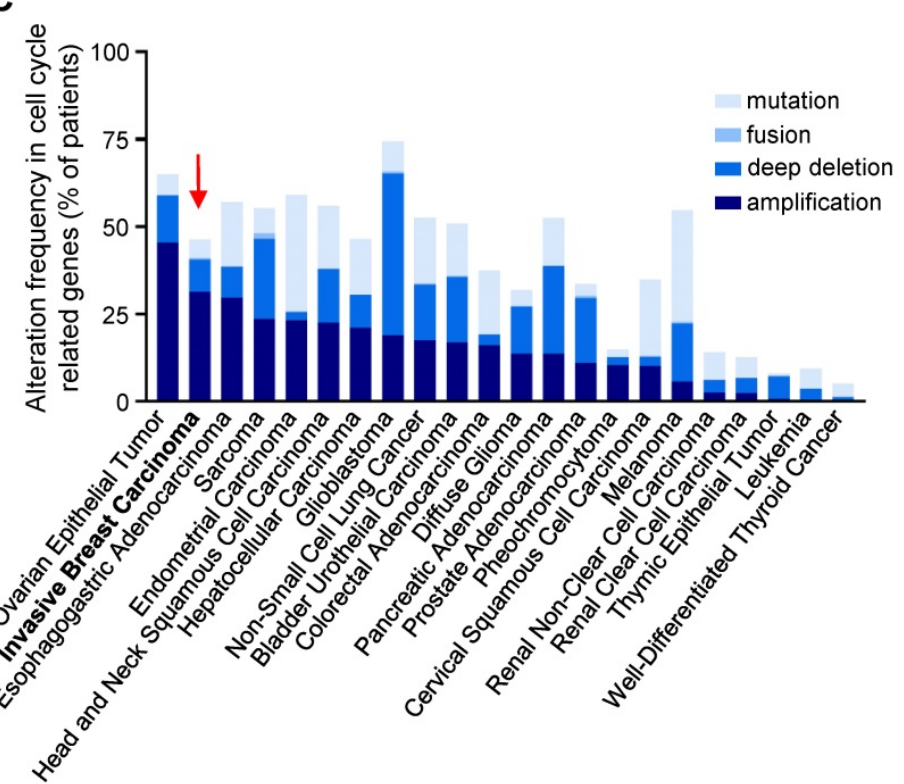

B

\begin{tabular}{|l|}
\multicolumn{2}{c|}{ KEGG 2019} \\
\hline Cell cycle \\
\hline p53 signalling pathway \\
\hline Oocyte meiosis \\
\hline ECM-receptor interaction \\
\hline Protein digestion and adsorption \\
\hline Progesterone-mediated oocyte.. \\
\hline Focal adhesion \\
\hline Pyrimidine metabolism \\
\hline Amoebiasis \\
\hline Drug metabolism \\
\hline $0 \quad 100 \quad 150 \quad 200 \quad 250$ \\
\multicolumn{1}{c}{ Combined score } \\
\hline
\end{tabular}

WikiPathways 2015 Gastric cancer network 1 (H.sap) RB in Cancer (H.sap) Cell Cycle (H.sap) Catalytic cycle of mamm..(H.sap) Neurotransmitter Clear.. (H.sap) Inflammatory Response..(M.mus) Inflammatory Response..(H.sap) Fluoropyrimidine Activity (H.sap) Gastric cancer network 2 (H.sap) miRNA regulation of DNA...(M.mus)

D

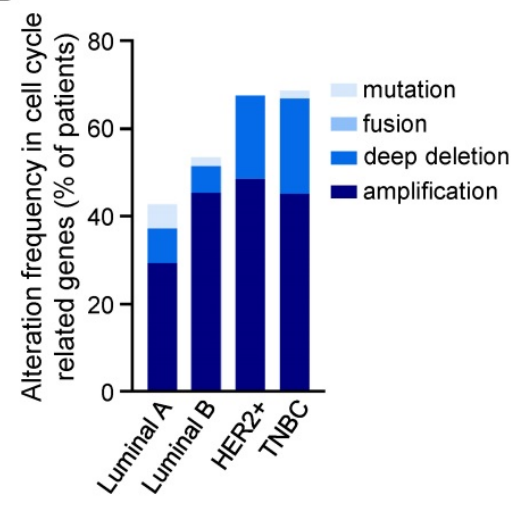

Figure 1. Cell cycle regulators are enriched and altered in breast cancer. (A,B) Human breast tumours are enriched in cell cycle regulators. (A) Analysis from three breast cancer databases (GSE42568, GSE45827, and GSE54002) delineated 97 common differential expressed genes, as reported in [23]. In the STRING network, proteins are represented by the nodes, and interaction between proteins by edges. Out of the 97 genes, 23 are involved in cell cycle regulation according to the Reactome database (red circles; see Table S5). See Figure S1 for high magnification. (B) Enrichment pathway analyses of the 97 differentially expressed genes using the Enrichr software, according to KEGG 2019 and WikiPathways 2015 databases, ordered according to the combined score. The 10-top ranked enriched pathways are shown and highlight signals involved in cell cycle regulation (red arrowhead). (C,D) Analysis of the Pan-Cancer Atlas cohort (TCGA database) of cell cycle-related gene alterations. Only patients with mutations and copy number variations data were selected for this analysis. Information regarding cell cycle-related gene alterations have been extracted with the use of cBioPortal for Cancer Genomics. The histograms report frequency (\% of patients) of cell cycle-related gene alterations in different cancer types (C) and in the four breast cancer sub-types (D). Alterations include mutation, gene fusion, deep deletion, and amplification. Note that breast cancer (indicated by a red arrow) ranks second among all cancer types analysed, sorted by amplification events. 

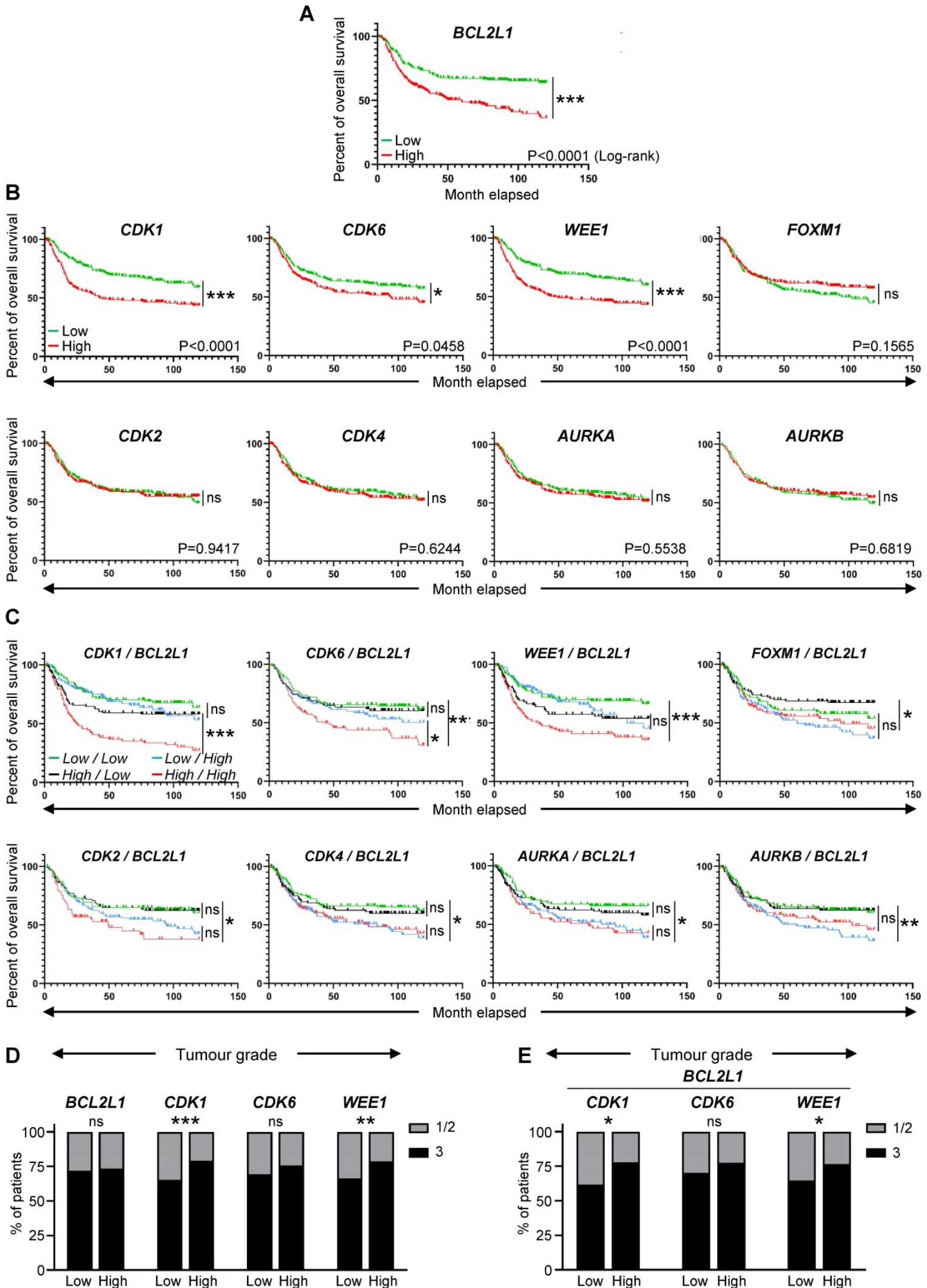

Figure 2. High expression levels of $B C L 2 L I, C D K I, C D K 6$, and WEEI correlate with lower survival rate of TNBC patients. (A-C) Analysis of publicly available microarray data from 580 TNBC patients (GSE31519 Affymetrix Human Genome U133A Array). Kaplan-Meier curves reporting the probability of the overall survival of human TNBC patients according to the expression levels of the BCL2LI gene, encoding BCL-XL (A), specific cell cycle regulators (CDKI, CDK2, CDK4, CDK6, WEEI, FOXMI, AURKA, and $A U R K B$ ) alone (B) or in combination with $B C L 2 L I(\mathbf{C})$. In these combinations (C), the expression levels indicated correspond to those of the cell cycle regulators and $B C L 2 L I$, respectively. The median of each gene expression levels was used as a threshold to segregate high versus low expressers. $P$ values were computed using the Logrank (Mantel Cox) test. (D,E) Tumour grades reported in patients with high or low levels of either single (D) or combined (E) indicated genes. Tumour grades were defined according to standard pathological scores (1-3; 3 corresponds to the most aggressive grade). Note that the $B C L 2 L I$ gene codes for BCL-XL, and that the AURKA and the $A U R K B$ genes encode the Aurora Kinase $A$ and the Aurora Kinase $B$, respectively. ns: not significant; $* P<0.05$; $* * P<0.01$; *** $P<0.001$. 
A MODELING HETEROGENEITY and DRUG RESISTANCE

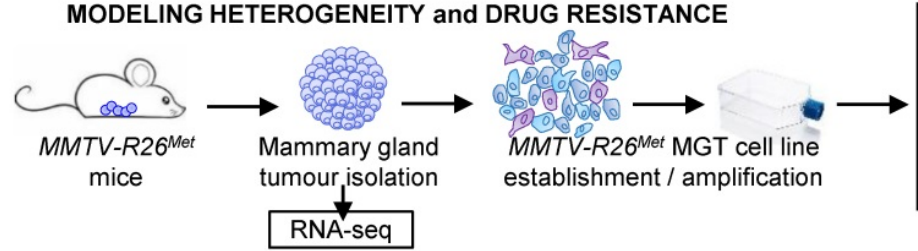

Explore specific vulnerability in targeting cell cycle regulators :

- Proteomic profiling

- Educated-guess drug screen

- Biochemistry
B RNA-seq : WikiPathways Cell Cycle C

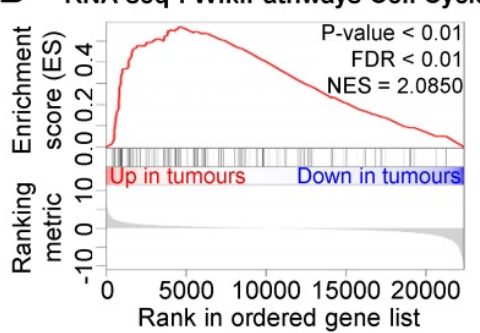

E
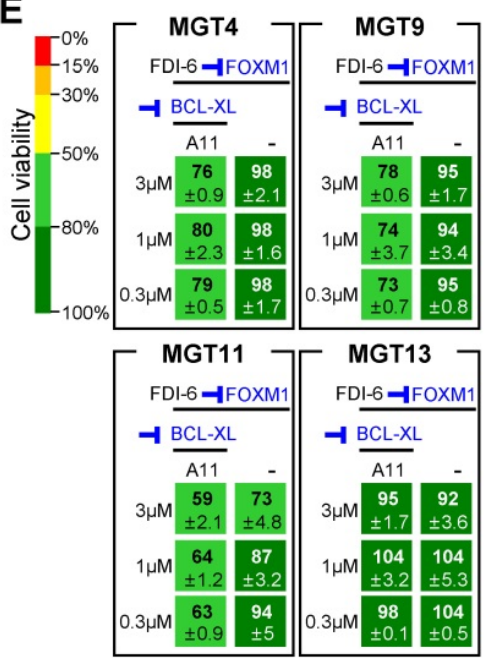

C

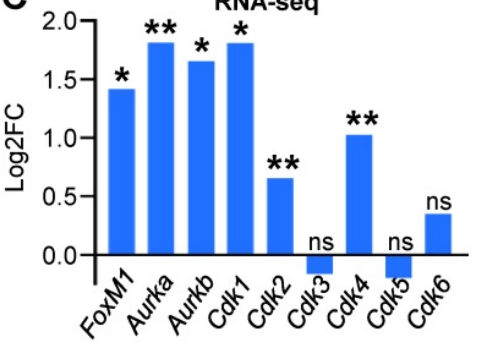

$\mathbf{F}$

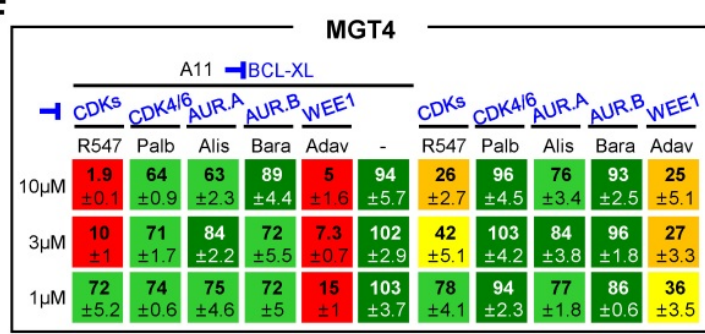

D

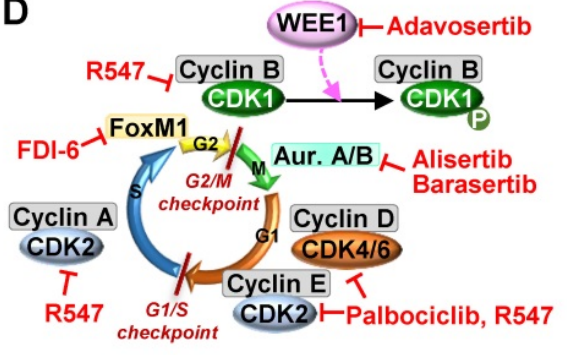

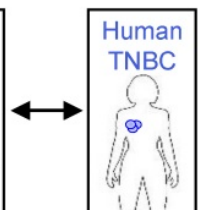

uman

1,
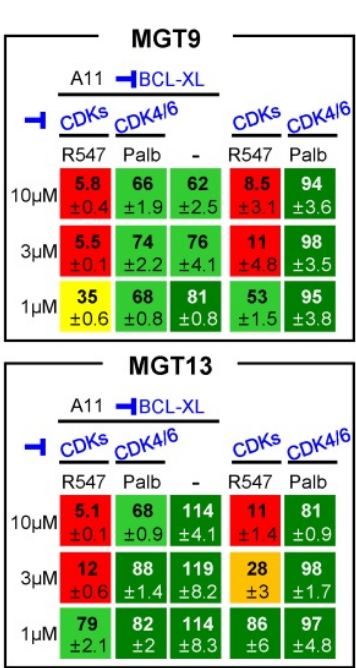

G

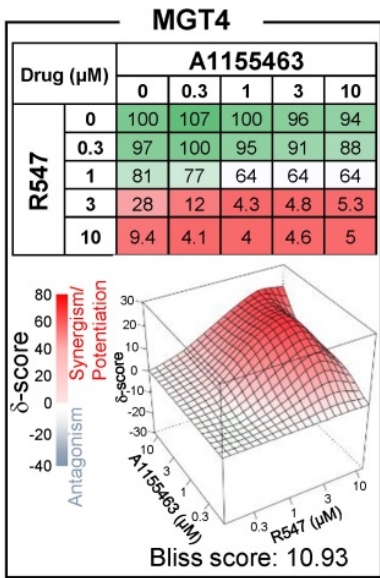

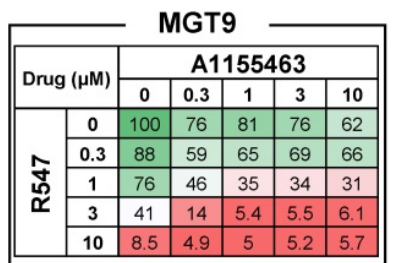
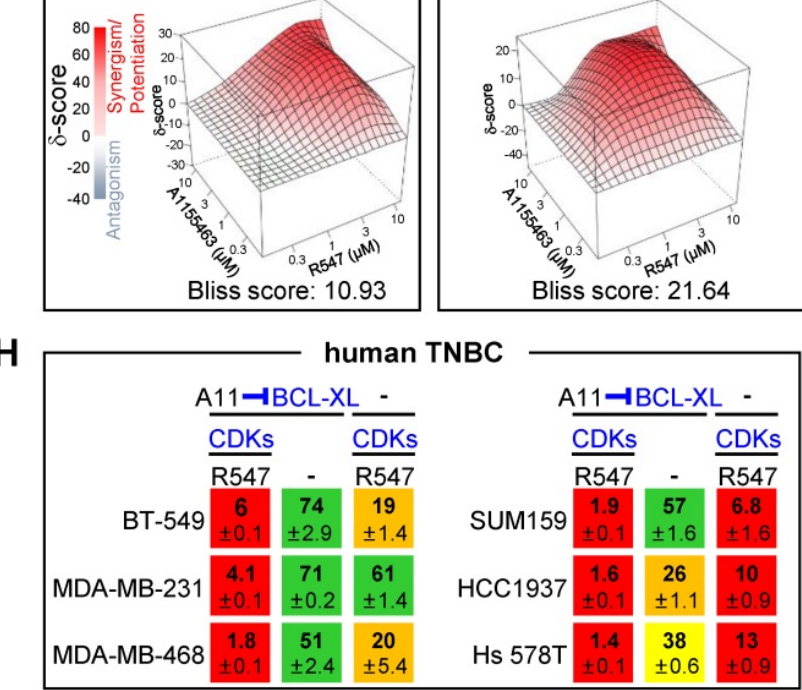
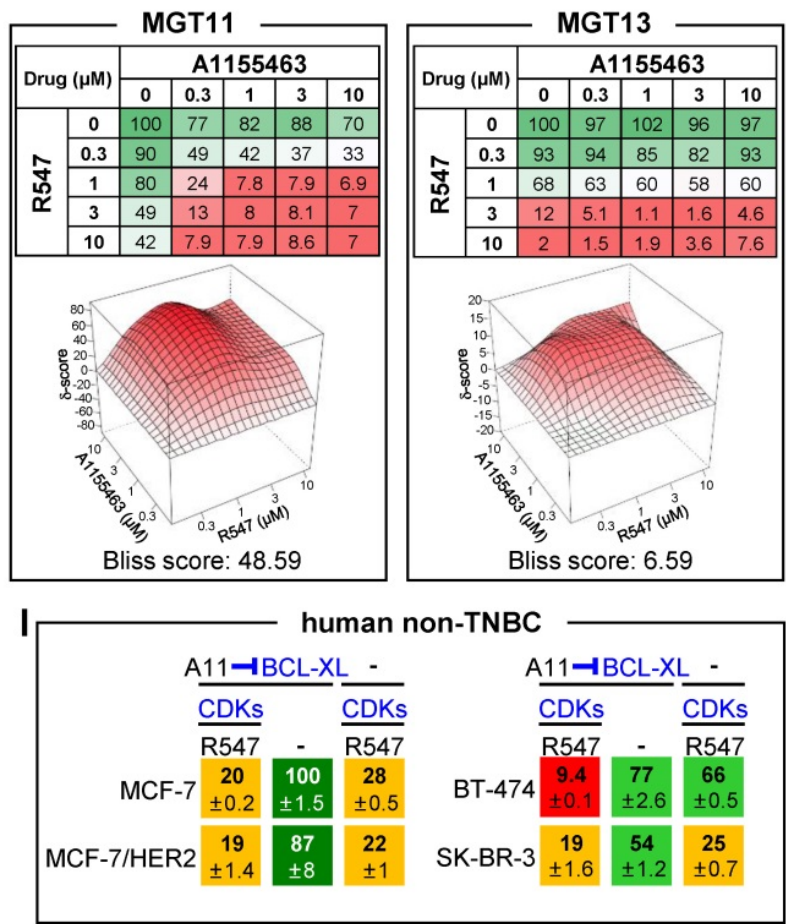

Figure 3. Combined inhibition of BCL-XL and CDK1/2/4 is deleterious for MMTV-R26Met and human TNBC cell lines. (A) Scheme illustrating the strategy used in this study to explore the vulnerability of TNBC cells to the inhibition of specific cell cycle regulators in combination with BCL-XL targeting. RNA-seq studies were performed 
on MMTV-R26Met tumours. Proteomic profiling, "educated-guess" drug screens and biochemical analyses were carried out on cell lines established from the MMTV-R26Met TNBC mouse model treated with drugs targeting cell cycle regulators together with BCL-XL. (B) GSEA enrichment performed, using the WikiPathways database, with cell cycle geneset on MMTV-R26Met tumours versus controls. The barcode plot indicates the position of a member of this geneset in the ranked list. Red and blue colours represent up- or downregulated genes in the MMTV-R26Met tumours versus the controls, respectively. NES: normalized enrichment score; FDR: false discovery rate. (C) Histogram depicting upregulation of FoxMl, Aurka, Aurkb, Cdkl, Cdk2, and Cdk4 transcript levels (as Log2FC; from RNA-seq data) in MMTV-R26Met tumours versus control tissues. Note that Cdk3, Cdk5, and Cdk6 mRNA levels were similar to controls. (D) Scheme illustrating key cell cycle regulators and their inhibitors used in this study. Their position within the cell cycle corresponds to the phase in which they act. (E) Effects of FOXM1 inhibition (FDI-6, 0.3-3 $\mu \mathrm{M}$ ) combined with BCL-XL targeting (A11: A1155463, 0.3 $\mu$ M) were analysed on the viability of the four tumorigenic MMTV-R26Met MGT cell lines. Numbers indicate the percentage of cell viability in the presence of drugs, compared to untreated cells (used as control). Percentages are reported using a green (high)-to-red (low) colour code (the scale depicted on the left is used as a reference in all studies). (F) Cell viability of the four MMTV-R26 $6^{\text {Met }}$ cell lines exposed to A1155463 (A11; targeting BCL-XL) alone or in combination with specific cell cycle regulators: R547 (targeting CDK1/2/4), Palbociclib (Palb; CDK4/6 inhibitor), Alisertib (Alis; targeting Aurora A), Barasertib (Bara; targeting Aurora B). Adavosertib (Adav; targeting WEE1) was used as control. (G) Top panel: Detailed matrix analysis of the MGT4, MGT9, MGT11 and MGT13 cell viability in response to A1155463 alone or in combination with R547. Bottom panel: Loewe plots and Bliss scores highlight synergism of the two drugs. Note that cell viability values with Al155463 alone are the same as reported in Figure 5a of [4], as both experiments were performed simultaneously, for direct comparison. (H-I) Cell viability of a panel of human TNBC (H) and non-TNBC (I) cell lines when exposed to A1155463 (1 $\mu$ M) and R547 ( $3 \mu$ M). In all figures, cell viability is presented as percentage of untreated cells (used as control). Values are expressed as means \pm s.e.m. At least three independent experiments were performed. For RNA-seq studies, statistical analyses were performed using "R". Adjusted P values are reported. ns: not significant; * P < 0.05; ** P<0.01. Aur.A: Aurora A; Aur.B: Aurora B; CDK: Cyclin-dependent kinase.

\section{TNBC cells are vulnerable to inhibition of selective cell cycle regulators following BCL-XL blockage}

The above findings, together with our recent studies uncovering the vulnerability of TNBC cells to combined WEE1 and BCL-XL targeting [4], drove us to explore the sensitivity of TNBC cells to inhibition of specific cell cycle regulators in combination with BCL-XL targeting. We addressed this issue using the $M M T V-R 26^{\text {Met }}$ TNBC model system, employing RNA-seq analysis, proteomic profiling, and cell viability assay as illustrated in Figure 3A. By Gene Set Enrichment Analysis (GSEA) of RNA-seq outcomes comparing MMTV-R26 $6^{\text {Met }}$ tumours $(\mathrm{n}=4)$ to control mammary gland tissues (MMTV-Cre; $\mathrm{n}=3)$, we found a striking enrichment in genes related to cell cycle regulation (Figure $3 \mathrm{~B}$ ), and to DNA replication and DNA damage pathways (Figure S3A-B, and Table S7). Specifically, we found significantly high mRNA levels of FoxM1 (a member of the Forkhead superfamily of transcription factors regulating a plethora of genes throughout the cell cycle to control DNA replication, mitosis, and cell proliferation), Aurka and Aurkb (cell cycle regulated kinases involved in microtubule formation and/or stabilization at the spindle pole during chromosome segregation), and Cyclindependent kinases $C d k 1, C d k 2$, and Cdk4 (which control progression through the cell cycle in concert with their cyclin regulatory subunits). Specific cyclin genes such as Cona2, Conb1, Ccnb2, and Cone1 encoding CYCLIN A2, B1, B2, and E1, respectively, were also upregulated in MMTV-R26 ${ }^{\text {Met }}$ tumours (see Table S8). Instead, comparable levels between tumours and normal mammary glands were found for $C d k 3, C d k 5$, and $C d k 6$ (Figure 3C).

In light of these results, we evaluated cell viability of mammary gland tumour (MGT) cell lines (MGT4, 9, 11, and 13) derived from the MMTV-R26 $6^{\text {Met }}$ model [4] when treated with inhibitors of various cell cycle regulators, acting during different phases, as illustrated in Figure 3D. Inhibition of either FOXM1 (with FDI-6), CDK4/6 (with Palbociclib), Aurora A or $B$ (with Alisertib or Barasertib, respectively) together with BCL-XL blockage (with A1155463) did not alter the viability of the MMTV-R26 $6^{\text {Met }}$ TNBC cells we tested (Figure 3D-F). We observed only a partial response in MGT11 cells following combined inhibition of BCL-XL with CDK4/6, Aurora A or Aurora $\mathrm{B}$ (Figure $3 \mathrm{~F}$ ). In contrast, combined targeting of CDK1/2/4 (with R547) and BCL-XL (with A1155463) was highly deleterious for all MMTVR26 $6^{\text {Met }}$ TNBC cells we tested (Figure $3 \mathrm{D}$ and F). Concomitant inhibition of CDK1/2/4 and BCL-XL was synergistic for 3 out of $4 M M T V-R 26^{\text {Met }}$ MGT cell lines, as shown by the Bliss score and the Chou-Talalay combination index score calculation (Figure 3G and S3C). These results were corroborated by the deleterious effect of CDK1/2/4 and BCL-XL co-targeting we observed in all six human TNBC cell lines tested (Figure $3 \mathrm{H}$ ). In contrast, we found only a modest effect on human non-TNBC cells (except for the BT-474 cells), similar to the non-tumorigenic MMTV-R26 ${ }^{\text {Met }}$ MGT2 cells (Figure 3I and S3D).

To further strengthen the relevance of targeting BCL-XL together with CDK1/2/4, we performed in vivo experiments by injecting orthotopically MDAMB-231 human TNBC cells into immunecompromised nude mice (Figure 4A). The effects of the A1155463 + R547 drug combination on tumour growth was evaluated overtime and at the end point of the experiment. Results show that mice treated with the drug combination had significant smaller tumours than those treated with the vehicle, as illustrated by measuring tumour volume overtime (Figure 4B), tumour volume and weight at the end point (Figure 4C-D, respectively). No significant side effects of the drug combination were observed by following the body weight of the mice during the whole procedure (Figure 4E). Collectively, these findings illustrate the vulnerability of TNBC cells to the inhibition of specific cell cycle regulators when BCL-XL is targeted. 
A
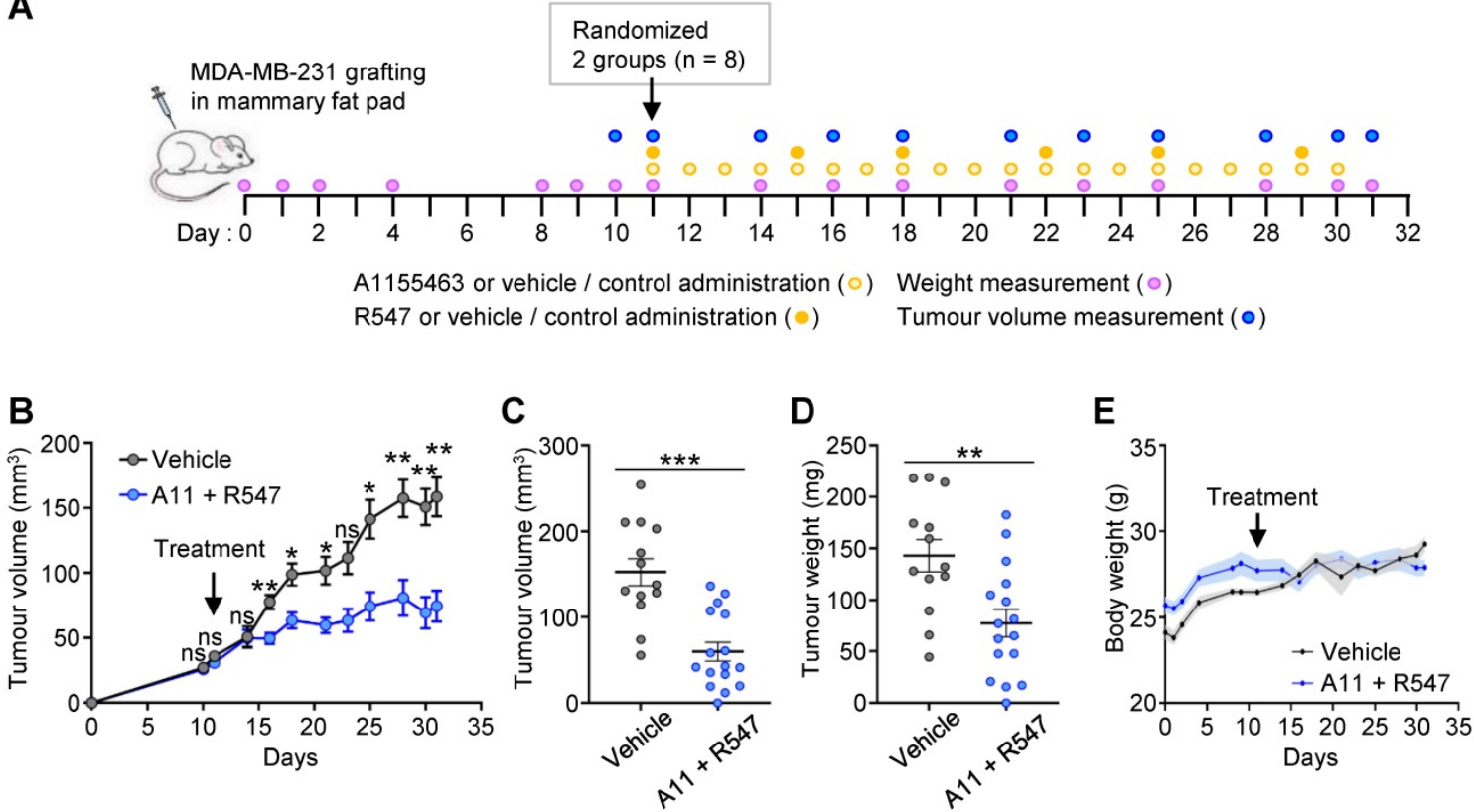

Figure 4. Combined A1155463 + R547 treatment interferes with tumour growth in orthotopic grafts. (A) Scheme illustrating orthotopic injection of MDA-MB-231 cells in the mammary fat pad of nude mice, frequency of drugs administration, mouse weight and tumour volume measurement. (B-D) Effects of A1 $155463+$ R547 treatment on tumour growth. (B) Evolution of tumour volume during the whole experiment. (C-D) Graphs reporting tumour volume (C) and tumour weight (D) at the end point of the experiment (day 31). (E) Graph showing the evolution of the body weight of mice during the whole procedure. No significant changes were observed, indicating that the dose of drugs used in vivo were not toxic. A1 1: A1 155463. Values are expressed as the mean \pm s.e.m. For B: two-way ANOVA statistical analysis was performed. For C and D: unpaired Student's t test was used. ns: not significant; $* \mathrm{P}<0.05 ; * * \mathrm{P}<0.01$; *** $\mathrm{P}<0.001$

\section{Combined BCL-XL and CDK1/2/4 inhibition interferes with cell cycle and survival signals, and triggers apoptosis}

We next examined, in the context of BCL-XL blockage (by A1155463), the molecular and biological consequences of CDK1/2/4 targeting (by R547) [24], and assessed specificity in alterations compared to WEE1 inhibition (by Adavosertib). Both drug combinations exhibited comparable IC50 in the four tumorigenic MMTV-R26 ${ }^{\mathrm{Met}}$ MGT cell lines (Figure S4). We first assessed the status of cell cycle regulators and observed a drastic downregulation of both RB protein expression and phosphorylation levels following CDK1/2/4 targeting (R547), in contrast to unchanged levels following WEE1 inhibition (Adavosertib) (Figure 5A-B, S3E, and S5). This is consistent with RB being a direct target of CDK2 and CDK4/6 [25]. FOXM1 phosphorylation levels were also severely reduced following CDK1/2/4 inhibition (Figure $5 \mathrm{~A}, \mathrm{~B})$. This is again consistent with FOXM1 being a direct target of CDK1 and CDK2 [26]. In contrast, WEE1 led to a slight increase in phospho-FOXM1 concurrent with an increase in CDK1 activity, as illustrated by loss of CDK1 phosphorylation (Figure $5 \mathrm{~A}, \mathrm{~B}$ and S5). Changes in p53 protein and phosphorylation levels were more pronounced following WEE1 inhibition than with CDK1/2/4 inhibition (Figure 5A and S5), although varying in relation to the MGT p53 status that we reported previously [4]. These findings were corroborated by a semi-quantitative RPPA proteomic profiling, a high-throughput antibody-based technique to analyse protein activities in signalling networks (Figure S3E, Table S9 and S10). Interestingly, we found a specific downregulation of Cyclin D3 levels following CDK1/2/4 inhibition (Figure 5C), correlating with downregulation of PP1 (Figure 5C), a phosphatase that stabilizes Cyclin D3 by keeping it in a dephosphorylated state [27]. No significant changes were observed in Cyclin B1, Cyclin D1, and Cyclin E1 levels (Figure S3F). Collectively, these findings illustrate that $\mathrm{CDK} 1 / 2 / 4$ inhibition has drastic consequences on cell cycle regulators we tested, not significantly exacerbated by BCL-XL targeting.

We then analysed the cell cycle effects of R547 alone or in combination with BCL-XL targeting (with A1155463), by following the distribution of cells in the cycle phases through flow cytometry. In the presence of R547, we observed a decrease in the percentage of cells in $S$ phase accompanied by an accumulation in G2 (Figure 5D, S3G, and Table S11). These results indicate a cell cycle blockage at G1-S and G2-M transitions, as previously reported [24]. Combined A1155463 + R547 treatment led to an accumulation of cells in G1, consequently reducing the percentage of cells in G2 compared to R547 monotherapy, in agreement with previously reported actions of BCL-XL on cell cycle regulation $[28,29]$. 
A

$\begin{array}{lllll}\text { MGT4 } & & \text { MGT9 } \\ \text { A11 A11 } & \text { A11 A11 }\end{array}$

A11 R547 Adav R547 Adav

$\begin{array}{r}\text { MGT11 } \\ \hline \text { A11 A11 }\end{array}$
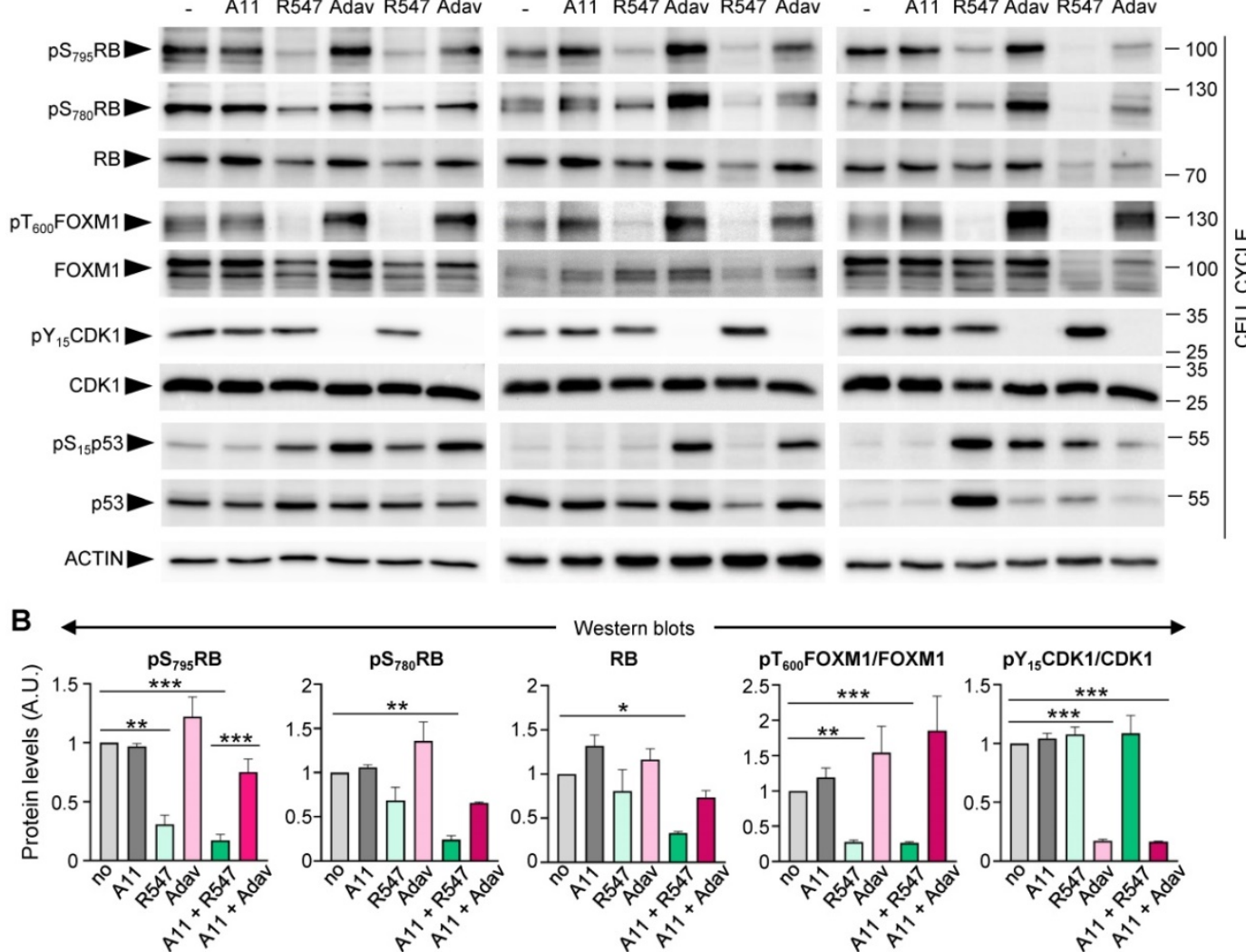

$----$
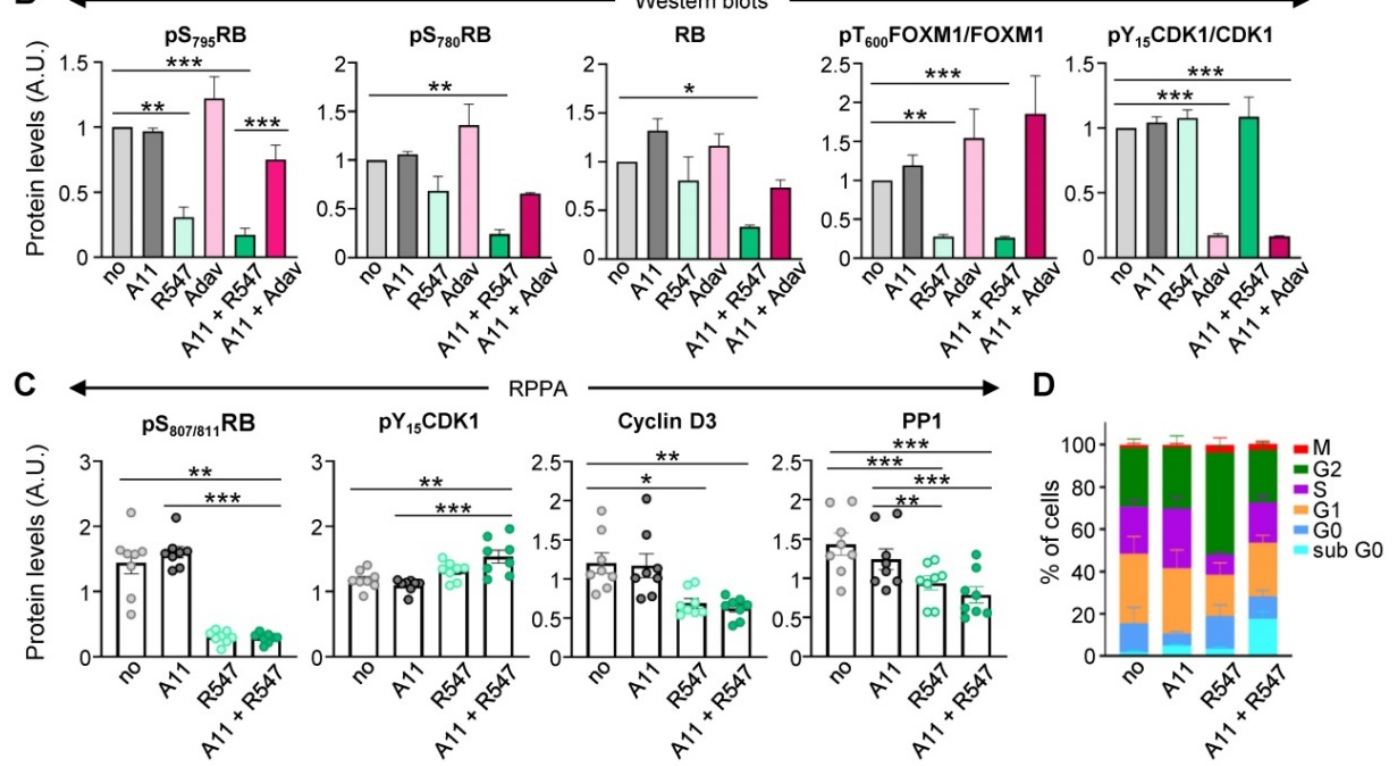

Figure 5. Inhibition of BCL-XL together with CDK1/2/4 perturbs cell cycle regulators. (A) Western blots performed on MGT4, MGT9, and MGT11 cells treated for

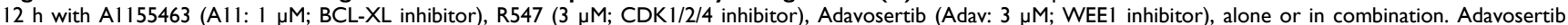
treatment was used for comparative studies. ACTIN, together with Ponceau (see the non-edited gels in Supplementary data), was used as loading control in all studies. Molecular weight markers ( $\mathrm{kDa}$ ) are indicated on the right in all western blots. (B) Protein levels estimated by densitometric analysis (using Image J) of western blots. Mean values obtained with the three MGT cell lines are shown as fold of control (not treated cells). Statistical analyses were done using ANOVA followed by Tukey test. (C) Changes in the expression/phosphorylation levels of the reported proteins in all four tumorigenic MMTV-R26 Met cell lines (MGT4, MGT9, MGT11, MGT13), either untreated or treated with the indicated drugs, based on the RPPA analysis (Table S9). (D) Histogram reporting the percentage of cells in each phase of the cell cycle when treated with the indicated drugs (for $12 \mathrm{~h}$ ), compared to non-treated cells (no). Distribution of cells in the different cell cycle phases was determined by flow cytometry using Pl and Ki67 staining. Three independent experiments were done. Statistical analyses were performed by one-way (B-C) or two-way (D) ANOVA followed by Tukey test, and are reported in Table S11. * P $<0.05 ; * *$ P $<0.01 ; * * * \mathrm{P}<0.001$. A.U.: arbitrary units.

We next explored the consequences of BCL-XL + CDK1/2/4 inhibition on regulators of cell survival and DNA damage and compared them with those linked to the BCL-XL + WEE1 targeting that we previously reported [4]. We found a drastic decrease in anti-apoptotic XIAP and MCL-1 protein levels in cells treated with BCL-XL + CDK1/2/4 targeting, accompanied by increased cleavage of Caspase 3, Caspase 7, and PARP, as shown by western blot (Figure 6A-B, and S5) and RPPA (Figure 6C and Table S9) analyses. Moreover, the combined treatments led to a high extent of DNA damage in cells, as revealed by the levels of $\gamma \mathrm{H} 2 \mathrm{AX}$ (a histone variant considered as a double-strand break sensor), by western blot (Figure 6A) and immunocytochemistry (Figure 6D). The high levels of DNA damage were accompanied by a downregulation of protein and/or phosphorylation levels of both ATM and ATR in cells treated with BCL-XL + CDK1/2/4 inhibitors, in contrast to unchanged, or a slight upregulation following BCL-XL + WEE1 blockage (Figure 6A,B), indicating a deficiency in the DNA damage detection mechanism 
induced by the A1155463 + R547 drugs. Furthermore, this combined BCL-XL + CDK1/2/4 treatment did not affect the percentage of phospho- $\mathrm{S}_{10}$ Histone $\mathrm{H} 3$ (pH3)-positive cells and did not trigger any mitotic catastrophe events in comparison to those observed in BCL-XL + WEE1 treated cells (Figure 6E-F). This agrees with the blockage of CDK1 by R547, therefore promoting cell cycle exit, in contrast to premature entry into mitosis observed with BCL-XL + WEE1 blockage (Figure $6 \mathrm{~F}$ ), as we showed in [4]. Together, these results illustrate that $\mathrm{CDK} 1 / 2 / 4$ inhibition together with BCL-XL targeting is as detrimental as BCL-XL + WEE1 blockage for TNBC cells, with similar perturbations of cell survival regulators, while having distinct effects on cell cycle and DNA repair components.

A

A

$\begin{array}{lll}\text { A11 A11 } & \\ & \text { A14 }\end{array}$

\begin{tabular}{ccc} 
MGT9 & \\
\hline A11 & A11 \\
- A11 R547 Adav R547 Adav
\end{tabular}

MGT11

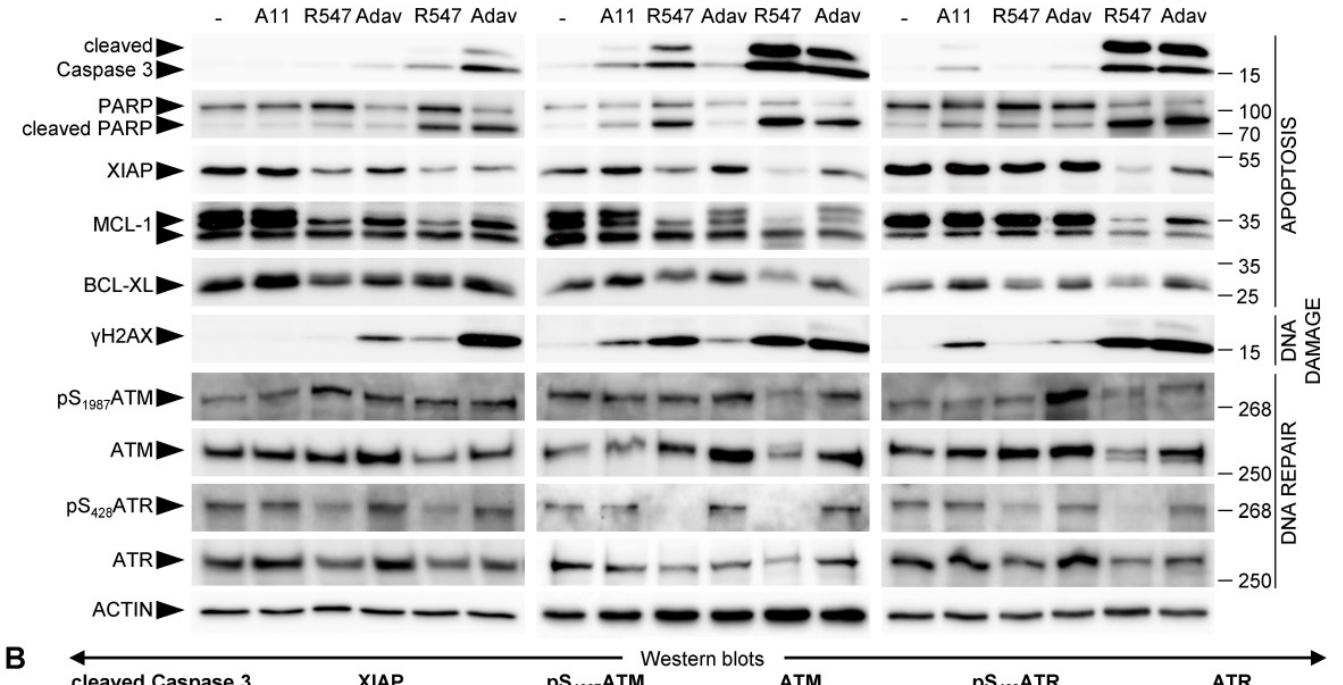

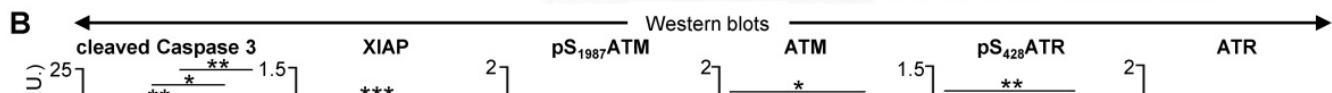

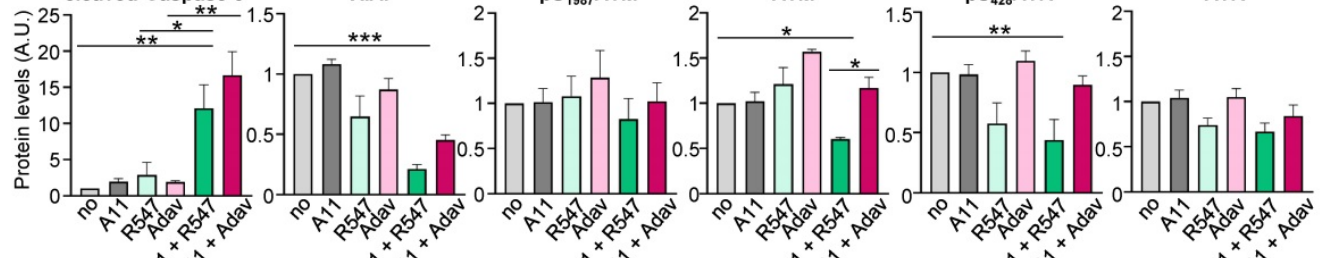

C

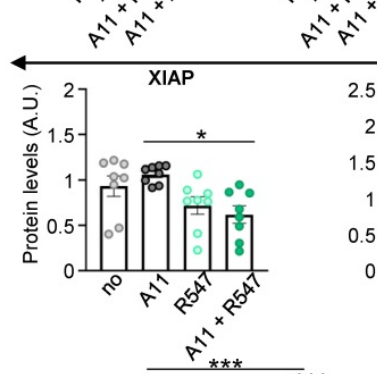

D

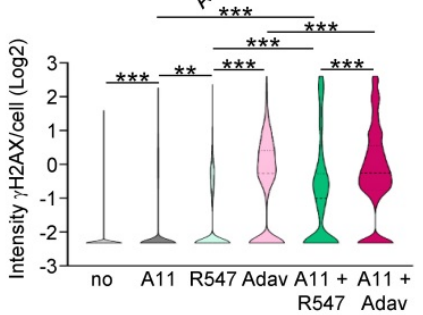

E
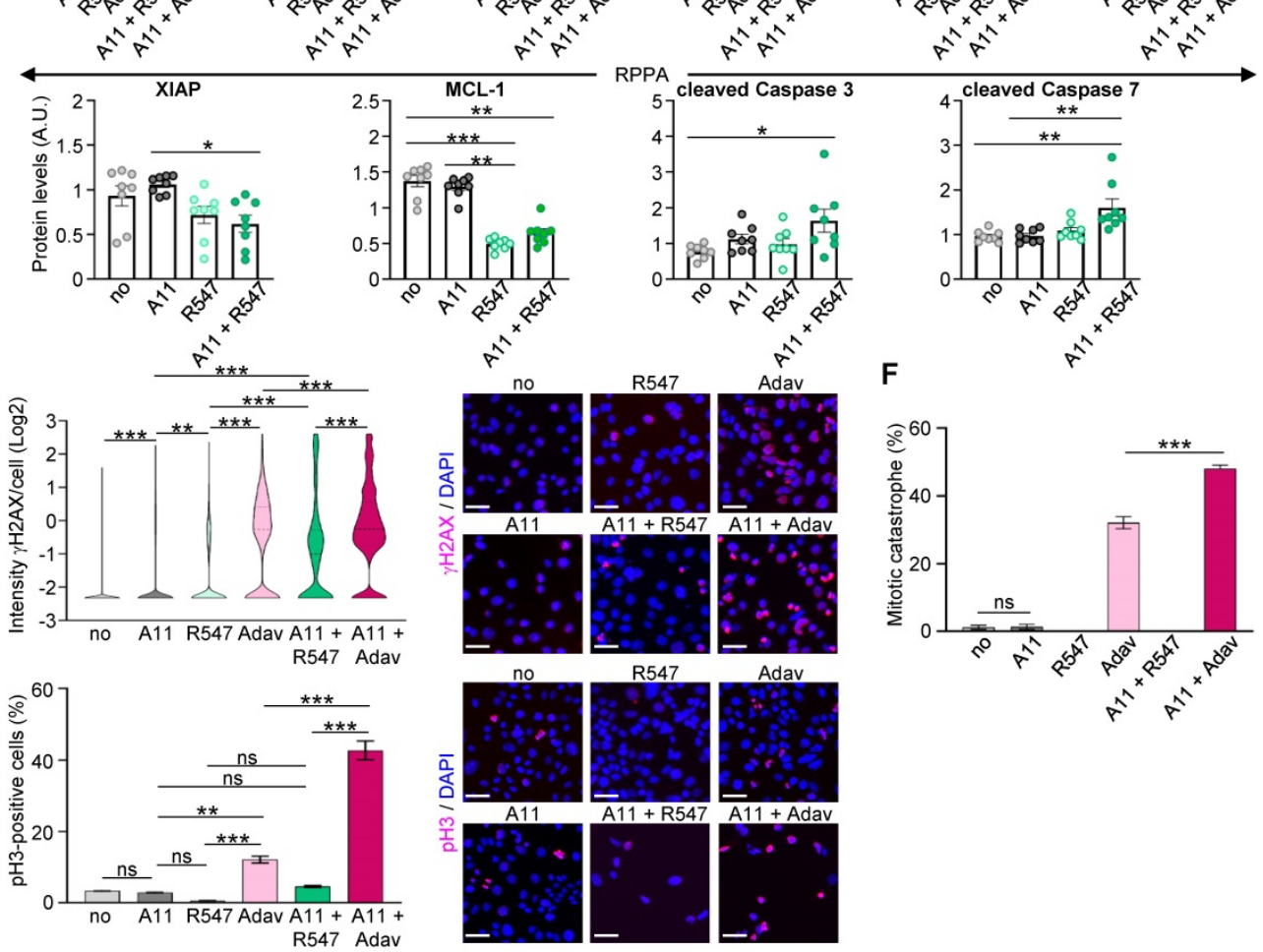

Figure 6. Co-targeting of BCL-XL and CDK1/2/4 induces DNA damage, interferes with survival signals, and triggers apoptosis. (A) $M M T V-R 26$ Met cells were treated for $12 \mathrm{~h}$ with either A1155463 (A1 1: $1 \mu \mathrm{M}$; BCL-XL inhibitor), R547 (3 $\mathrm{M}$; CDK1/2/4 inhibitor) or Adavosertib (Adav: $3 \mu \mathrm{M}$; WEE1 inhibitor), alone or in combination, 
then subjected to western blot analysis. Note that R547 alone increased DNA damage in the MGT9 cell line, which exhibits the highest p53 levels as we reported in [4]. We underline that the ACTIN panels are the same as in Figure 5 as western blots were performed simultaneously. (B) Densitometric analysis (using Image J) of western blots depicting protein levels. Mean values obtained with the three MGT cell lines are shown as fold of control (untreated cells). (C) Graphs depicting changes in levels of anti-apoptotic proteins (XIAP and MCL-1) as well as apoptosis markers (cleaved Caspase 3 and cleaved Caspase 7) in the four tumorigenic MMTV-R26Met cell lines following treatment with the indicated drugs, based on the RPPA analysis (Table S9). (D-F) DNA damage and mitotic catastrophe analysis. MGT11 cells were treated or not with A1155463 (0.3 $\mu$ M), R547 $(3 \mu \mathrm{M})$, Adavosertib $(3 \mu \mathrm{M})$, or in combination. (D) Immunostaining with anti-yH2AX antibodies (to assess DNA damage) was performed after $12 \mathrm{~h}$ of treatment. The violin plot depicts the number of cells according to their $\mathrm{yH} 2 \mathrm{AX}$ staining intensity. Representative images of $\mathrm{\gamma H} 2 \mathrm{AX}$ immunostaining (red) are shown on the right. (E) Cells treated for 16 $\mathrm{h}$ with the indicated drugs were immunostained with anti- $\mathrm{pH} 3$ antibodies. The graph reports the percentage of cells in mitosis ( $\mathrm{pH} 3-\mathrm{positive} \mathrm{cells)} \mathrm{versus} \mathrm{the} \mathrm{total} \mathrm{number} \mathrm{of} \mathrm{cells.}$ Representative images of $\mathrm{pH} 3$ immunostaining (red) are shown on the right. (F) Histogram reporting the number of mitotic catastrophe, (revealed by anti-pH3/a-Tubulin (microtubules) immunostaining) in treated cells. Mitotic catastrophe was analysed in cells in metaphase and anaphase among the pH3-positive cells. In contrast to WEE1 targeting (with Adavosertib), as previously reported [4], R547 does not induce mitotic catastrophe. In all experiments, DAPI was used to counterstain the nuclear DNA. Three independent experiments were performed. For multiple comparisons, statistical significance was assessed by One-way ANOVA followed by Tukey test. ns: not significant; $*$ P $<$ $0.05 ; * * \mathrm{P}<0.01$; *** $\mathrm{P}<0.001$. Scale bar: $50 \mu \mathrm{m}$

\section{Combined BCL-XL and CDK1/2/4 inhibition leads to downregulation of RTK and AKT signalling}

To obtain further insights on signalling changes occurring in cells treated with BCL-XL + CDK1/2/4 inhibition and associated with their death, we bioinformatically explored RPPA outcomes on expression and/or phosphorylation levels of 426 proteins (Figure S3E, Table S9 and S10). First, we compared alterations occurring with single (BCL-XL or CDK1/2/4) versus combined BCL-XL + CDK1/2/4 targeting. We found that combined BCL-XL + CDK1/2/4 inhibition caused 177 alterations: 73 of which were already present following CDK1/2/4 inhibition (Figure 7A). Only 4 alterations were found following BCL-XL targeting (Figure 7A). Interestingly, protein-protein interaction network analysis, using the STRING tool, highlighted three main enriched pathways: RTK signalling, AKT signalling, and cell cycle/DNA damage (Figure 7B and S6). Furthermore, we found that CDK1/2/4 targeting leads to alteration of RTK signalling (Figure 7C: blue), AKT signalling (orange), cell cycle regulators (green), and components of DNA damage/repair and apoptosis (yellow). Notably, the combined treatment exacerbated perturbation of AKT signalling and DNA damage/apoptosis effectors (Figure 7C).

We then compared signalling changes linked to BCL-XL + CDK1/2/4 inhibition with those occurring in cells following BCL-XL + WEE1 blockage. The top ranked signalling alterations observed specifically upon BCL-XL + CDK1/2/4 inhibition were mainly signals involved in RTK and/or AKT pathways (e.g. downregulation of: phospho-MET, phospho-HER2, phospho-AKT, phospho-NDRG1, phospho-SHP2, STAT3), while BCL-XL + WEE1 targeting mainly impacted regulators of cell cycle and DNA repair (Figure 7D). DNA damage/repair/apoptosis deregulation was seen with both combinatorial settings (Figure 7D).

To further examine the mechanisms underlying the effects of these drug combinations, we performed a GSEA using all signals from the RPPA outcomes from cells treated with BCL-XL + CDK1/2/4 versus
BCL-XL + WEE1 inhibitors. Using the Reactome database, we found that gene-sets related to RTK signalling and second messengers, as well as PI3K/ AKT signalling, were among the most significant deregulated signals in $M M T V-R 26^{\text {Met }}$ MGT cells treated with BCL-XL + CDK1/2/4 inhibitors (Figure $7 \mathrm{E})$. This approach delineated a possible mechanism underlying combined BCL-XL plus CDK1/2/4 targeting, showing a pronounced alteration of RTK and AKT signalling.

Interestingly, it has recently been shown that CDK1, together with Aurora kinase, ensures RTK storage by suppressing endosomal degradation and recycling pathways [30]. We therefore further investigated the striking correlation between BCL-XL + CDK1/2/4 inhibition and the downregulation of RTK and ATK signalling. Western blot results revealed a consistent downregulation of expression and/or phosphorylation levels of several proteins, including MET, GAB1, ERKs, and AKT in cells with BCL-XL + CDK1/2/4 inhibition, although with slightly different intensities among the MMTV-R26 $6^{\text {Met }}$ MGT cells (Figure 8A and S5). These downregulations were further confirmed by performing kinetic studies (Figure 8B). Additionally, RPPA analysis showed downregulation of protein expression and/or phosphorylation levels of AKT, RICTOR, and FOXO3a following BCL-XL + CDK1/2/4 inhibition (Figure 8C).

Such correlation between BCL-XL + CDK1/2/4 inhibition and RTK and ATK downregulation was further explored in human TNBC cell lines. By comparing MET protein levels in three human TNBC (MDA-MB-231, SUM159, and Hs578T) and three non-TNBC (SK-BR-3, MCF-7, and BT-474) cell lines, we observed strong MET expression in all three TNBC cell lines analysed (Figure 8D). This correlates with higher MET expression levels in human TNBC patients compared to other breast cancer subtypes (Figure 8E). Assessing by western blots the effects on RTK signalling of either single or combined A1155463 and R547 drugs, we found a consistent cleaved Caspase 3 following combined treatment in the three human TNBC cell lines (Figure 8F). This was accompanied by a drastic downregulation of MET 
levels and of AKT expression and phosphorylation (Figure $8 \mathrm{~F}$ and 57 ). These signalling changes corroborate the drug combination effects on the viability of human TNBC cells reported in Figure 3H.
Collectively, these results illustrate a consistent depletion of MET and AKT in mouse $(\mathrm{n}=4)$ and human $(n=3)$ TNBC cell lines.
A

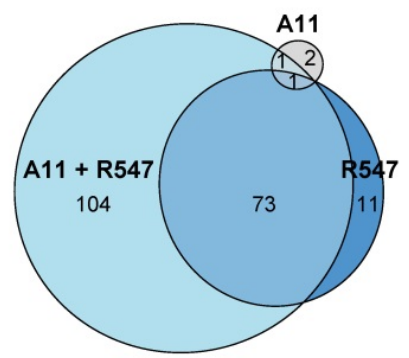

B

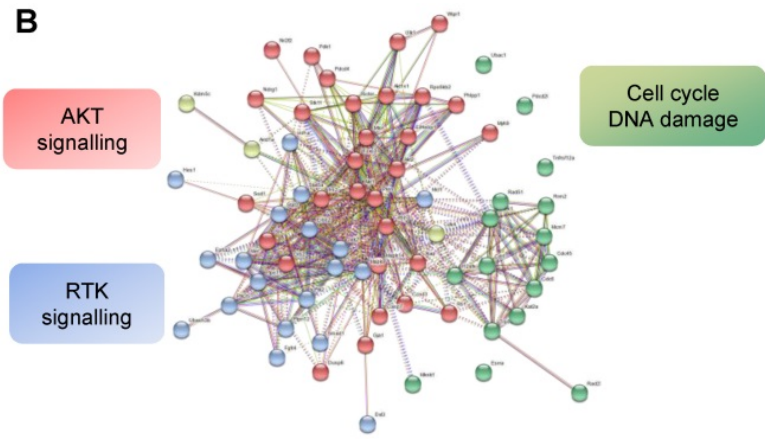

D
C

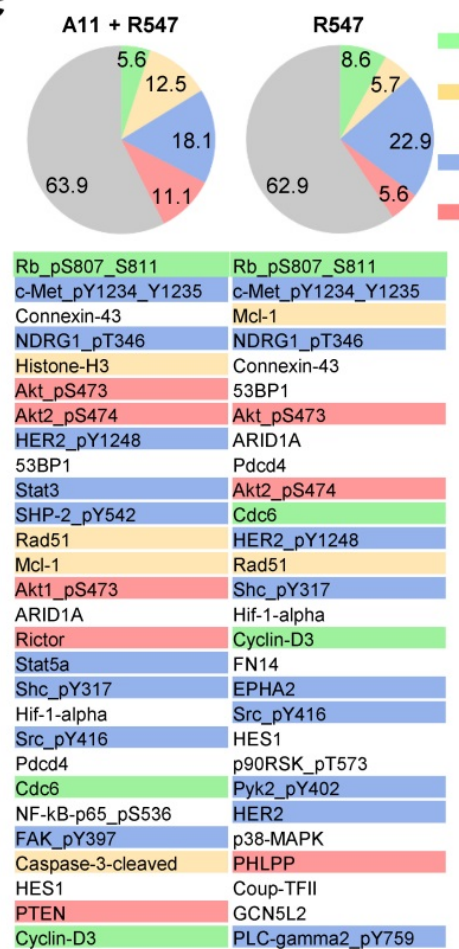

Cell cycle regulation

DNA damage / DNA repair / apoptosis

E

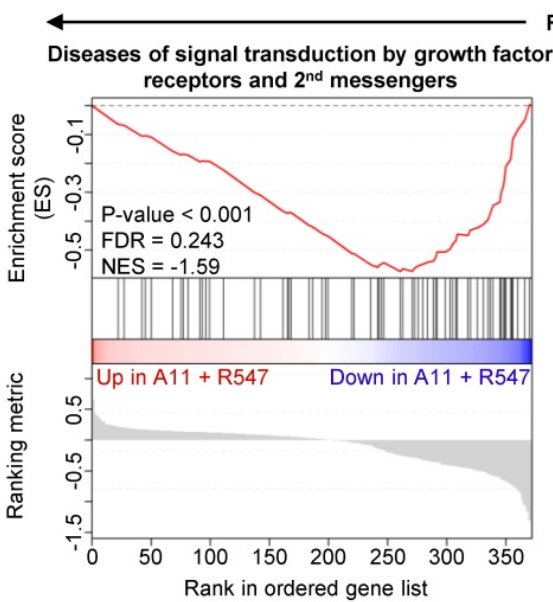

REACTOME

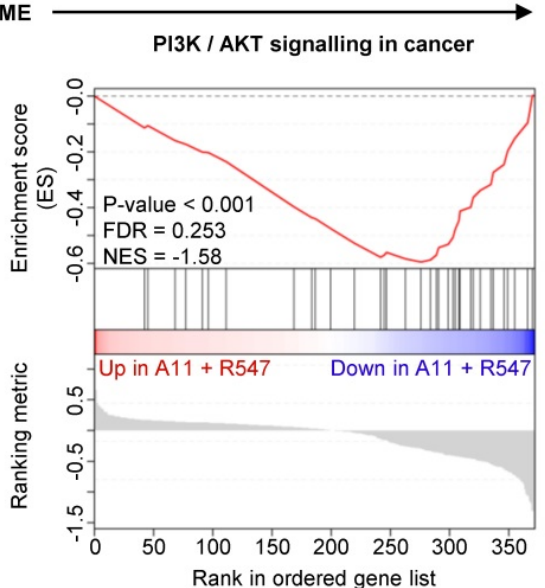

Figure 7. Proteomic analyses highlight downregulation of RTK and AKT signalling in the tumorigenic MMTV-R26Met cells when subjected to combined inhibition of BCL-XL and CDK1/2/4. (A) Euler diagram showing the number of dysregulated signals in all four MMTV-R26Met tumorigenic cells (MGT4, MGT9, MGT11, and MGT13) treated with the indicated drugs. The diagram was obtained by the Eulerr package in R (https://cran.r-project.org/package=eulerr). The area of each circle is proportional to the number of dysregulated signals. A cut-off of P value $<0.05$ was applied. (B) Projection of the dysregulated genes in cells treated with A1155463 + R547 onto the STRING 
protein-protein interaction network highlights 3 main clusters using the kmeans clustering method. Clustering was based on protein interaction. The cut-off value was predefined as $P$ value $<0.05$ and fold change $<-0.5$ or $>0.5$. See Figure $S 6$ for high magnification. (C) Diagrams representing the proportional distribution of signals in all four MMTV-R26Met cell lines treated with R547 alone or in combination with A1155463. Percentages are indicated. The cut-off value was predefined as $P$ value $<0.05$. The 30 -top ranked dysregulated signals are listed. (D) Euler diagram depicting the number of dysregulated signals in the MMTV-R26Met cells treated with either A11 + R547 or A11 + Adavosertib. A cut-off of $P$ value $<0.05$ was applied. The 10-top ranked dysregulated signals among either the specific (A11 + R547: 134; A11 + Adav: 21 ) or the common (A11 + R547/A11 + Adav: 45) signals are shown. For panels C and D: among signals, we highlighted RTK signalling (blue), AKT signalling (red), proteins involved in DNA damage/repair/apoptosis (yellow), and those in cell cycle regulation (green). (E) GSEA enrichment plots performed, using the REACTOME database, on all signals from A11 + R547 versus A11 + Adav data. Note that gene-sets related to RTK signalling and second messengers, as well as PI3K/AKT signalling, are in the top 10 downregulated Reactome pathways in BCL-XL + CDK1/2/4-treated MMTV-R26Met cells. The barcode plot indicates the position of a member of a gene set in the ranked list of proteins. Red and blue colours represent proteins up- or downregulated in A11 + R547 versus A11 + Adav, respectively. NES: normalized enrichment score; FDR: false discovery rate.

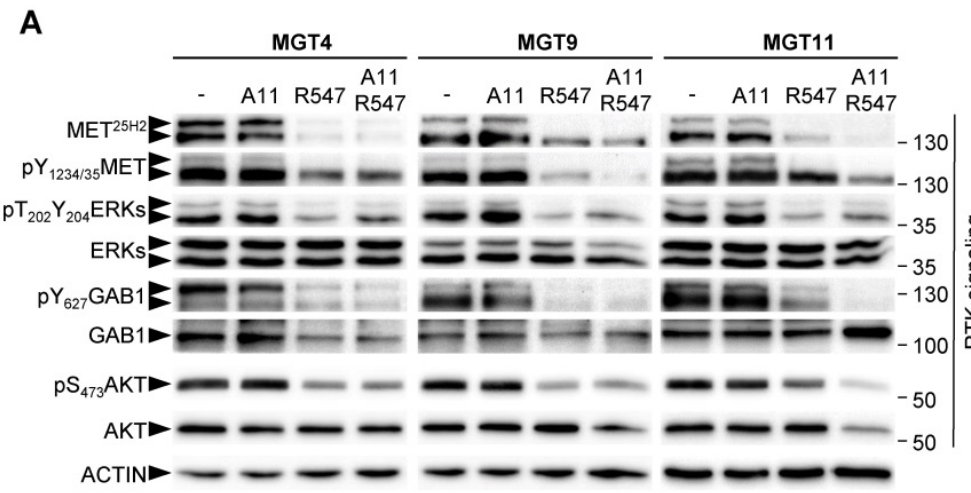

C
B

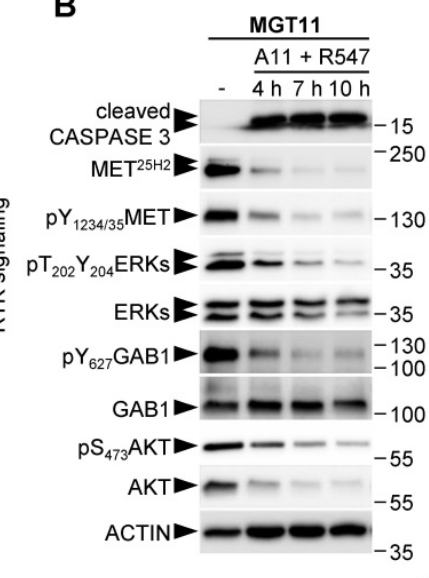

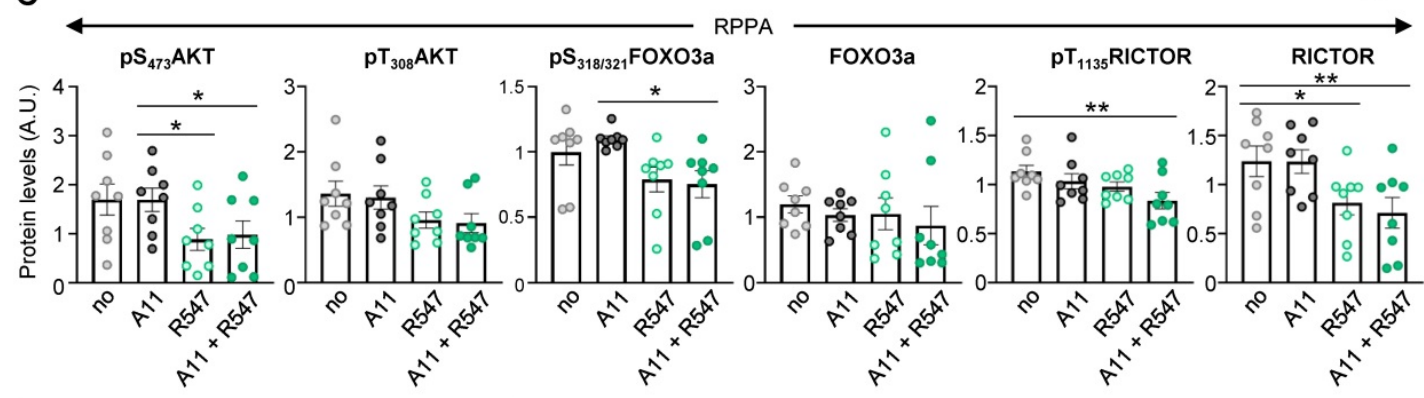

D

human BC cell lines TNBC non-TNBC

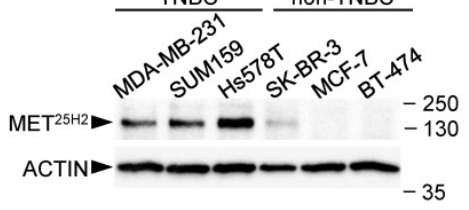

E
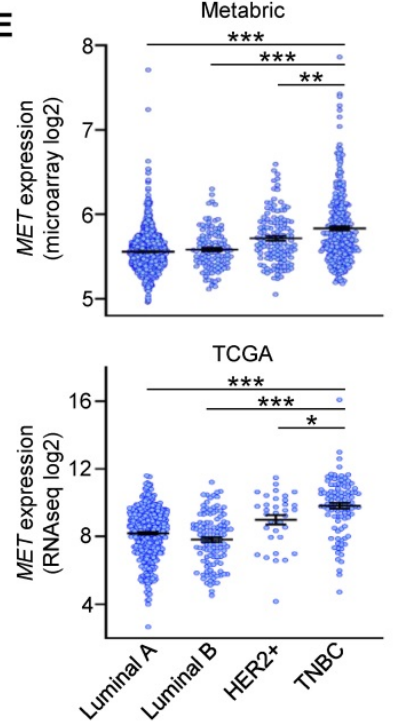

F

cleaved Caspase 3

MDA-MB-231 A11 ${ }^{\mathrm{A} 11}$ SUM159

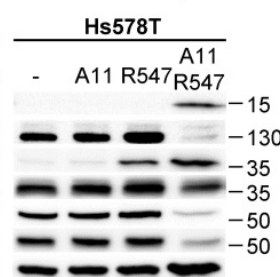

G

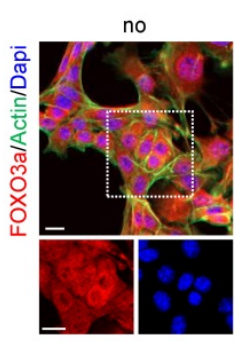

A11

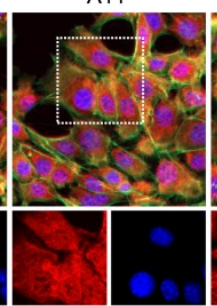

R547

$\mathrm{A} 11+\mathrm{R} 547$
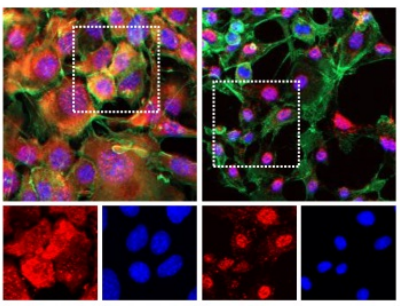

H

I

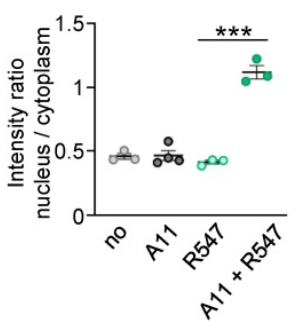

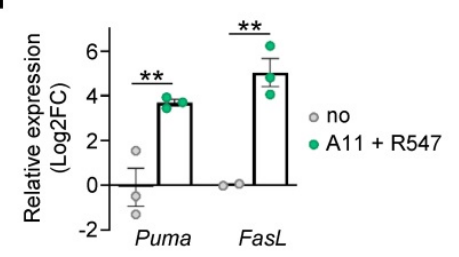

Figure 8. Combined BCL-XL and CDK1/2/4 inhibition leads to RTK/AKT signalling downregulation and FOXO3a nuclear retention with activation of apoptosis associated genes. (A) Western blots performed on MMTV-R26 Met MGT cells after a $12 \mathrm{~h}$ treatment with the indicated drugs. Note that the MET25H2 antibodies 
recognise both the endogenous mouse and the METtg (corresponding to the human sequence). (B) Kinetic analysis of changes in expression and/or phosphorylation levels of the indicated RTK signalling components after combined targeting of BCL-XL and CDK1/2/4. (C) Histograms reporting downregulation of expression and/or phosphorylation levels of AKT (on $\mathrm{S}_{473}$ and $\mathrm{T}_{308}$ ), FOXO3a, and RICTOR following BCL-XL and CDK1/2/4 inhibition, based on the RPPA outcomes. (D) Western blot analysis of total MET (MET25H2) expression levels in a panel of human breast cancer cell lines: TNBC (MDA-MB-231, SUM159, Hs578T) and non-TNBC (SK-BR-3, MCF-7, BT-474). (E) Graphs showing MET expression levels within the four breast cancer IHC-based subtypes from Metrabric (top panel, microarray) and TCGA (bottom panel, RNA-seq) databases. One-way ANOVA statistical analysis was performed. Bars represent mean +/- s.e.m. (F) Western blots performed on human TNBC cell lines (MDA-MB-231, SUM159, Hs578T) treated with A1155463 (A11: $1 \mu \mathrm{M}$; BCL-XL inhibitor), R547 (3 $\mu$ M; CDK1/2/4 inhibitor), alone or in combination. (G) MGT11 cells treated with the indicated drugs were immunostained with anti-FOXO3a (red) and phalloidin (to detect F-Actin, green). Note the nuclear retention of the FOXO3a protein when cells were treated with BCL-XL + CDK1/2/4 inhibitors (A11 + R547). DAPI (blue) was used to counterstain the nuclear DNA. The bottom panels depict split red and blue channels of the indicated areas. (H) Graph depicting the intensity ratio of nuclear versus cytoplasmic FOXO3a in cells when exposed to the indicated drugs. (I) RT-qPCR analysis of FOXO3a target genes in MGT11 cells treated with A11 + R547. Statistical significance was assessed by One-way ANOVA followed by Tukey test. $* P<0.05$; ** $P<0.01$; *** $P<0.001$. Scale bar: $20 \mu \mathrm{m}$.

The correlation between reduced phosphorylation of AKT and FOXO3a was particularly interesting, considering that AKT phosphorylates FOXO3a leading to its translocation from the nucleus to the cytoplasm, thus preventing its tumour suppressor function [31]. We therefore explored this aspect by performing immunocytochemistry to follow FOXO3a localisation in untreated and treated cells. We found a striking FOXO3a nuclear localization in cells treated with BCL-XL + CDK1/2/4 inhibition compared with cells either untreated or treated with single drugs (Figure 8G-H). This FOXO3a nuclear retention was accompanied by a transcriptional expression of PUMA and FasL, two FOXO3a target genes involved in apoptosis (Figure 8I). Together, these findings show that combined BCL-XL and CDK1/2/4 targeting, while perturbing cell cycle regulators, leads to a combinatorial depletion of survival and RTK/AKT signals, restoring FOXO3a tumour suppression actions.

\section{Discussion}

In this study, we provide evidence that combinatorial targeting of BCL-XL with CDK1/2/4 could be an efficient therapeutic approach for TNBC. This drug combination was potent across different TNBC subtypes, as demonstrated by its high efficacy in different human TNBC cell lines and in the heterogenous cell lines generated from distinct $M M T V-R 26^{\text {Met }}$ tumours. Such combination appears to be less effective on non-TNBC cells, based on the cell lines used in this study.

The alteration of cell cycle regulators in several types of cancer [10], including breast cancer, and the possibility to modulate their function, has fostered the interest to design treatment options targeting them, with some of them already being exploited in clinical trials [10]. The relevance of alterations of cell cycle regulators in breast cancer is strengthened by several -omics analyses and bioinformatic processing. For example, a recent study revisited breast cancer microarray datasets uncovering that differentially expressed genes are mainly enriched in cell cycle regulators [23]. Specifically, CDK4/6 oncogenic activation has been reported in luminal breast cancer, constituting one of the main tumorigenic drivers. In contrast, dysregulations associated with TNBC formation include $c-M y c$ activation, p53 mutations, PTEN-loss, and CDKs/Cyclins overactivation/ overexpression [10,32]. In this study, we have shown that TNBC patients with high levels of CDK1, CDK6, and WEE1, are characterised by a worse prognosis compared to those with low expression levels. Interestingly, we have also shown that poor TNBC prognosis is further exacerbated by high expression levels of BCL-XL.

The identification of vulnerabilities of cancer cells is particularly challenging for those types of cancer, like TNBC, highly heterogeneous and lacking major drivers to which cells are addicted to [33]. To uncover TNBC signalling vulnerabilities, relevant model systems mimicking disease characteristics are essential. We have recently reported the uniqueness of the MMTV-R26 $6^{\text {Met }}$ model, which recapitulates several features of TNBC including primary therapy resistance and marker intertumoral heterogeneity. This model has previously allowed us to uncover the potency of WEE1 targeting while lowering survival inputs through BCL-XL inhibition [4]. The present study further confirms the vulnerability of TNBC cells to targeting cell cycle regulators in the context of BCL-XL blockage.

In this regard, an intriguing aspect emerging from the present studies is that in the presence of $B C L-X L$, the specific subset of cell cycle regulator targeted determines the effect on TNBC cell viability. Specifically, TNBC cells are sensitive to combined inhibition of CDK1/2/4 by R547, but not of FOXM1, CDK4/6, Aurora A and B. FOXM1 is a transcription factor overexpressed in most solid tumours, such as breast, liver, prostate, colon, and pancreas [34]. This member of the Forkhead family regulates the expression of a large set of G2/M specific genes, and aberrant FOXM1 upregulation has been shown to be a key driver in cancer progression [26]. Our RNA-seq analysis showed an up-regulation of $C D K 1, C D K 2$, CDK4, FoxM1, Aurora A/B, but not of CDK3, CDK5, $C D K 6$, genes in $M M T V-R 26^{\text {Met }}$ tumours versus normal mammary glands, as reported in TNBC patients [35, 36]. Nevertheless, their inhibition together with BCL-XL targeting had no effect on the viability of the four MMTV-R26 $6^{\text {Met }}$ TNBC cell lines we examined. 
This apparent contradiction to previous studies [37] is likely explained by the fact that the effectiveness of FOXM1 or CDK4/ 6 targeting is conditioned by the set of molecular alterations present in cancer cells. Indeed, it has been shown that Palbociclib (a potent CDK4/6 inhibitor currently used in the clinic) in RB-proficient TNBC cells effectively potentiates subsequent treatments with chemotherapeutic agents like paclitaxel or cisplatin, although eliciting antagonist effects when simultaneously used [14, 38]. Furthermore, it has been shown that FoxM1 and Aurora A targeting overcomes TNBC paclitaxel resistance [39]. Our RPPA and biochemical studies showed that RB is hyper-phosphorylated in $M M T V-R 26^{\text {Met }}$ TNBC cells [4], although levels may not be sufficient to confer the sensitivity of cells to Palbociclib treatment. Alternatively, it is possible that the effects of Palbociclib in TNBC cells is conditioned by the drug used in combination. Here, we show that Palbociclib does not synergize with BCL-XL inhibition. The R547 drug we used in these studies is a potent ATP-competitive inhibitor of CDK1/2/4 [24]. The strong combinatorial effects of BCL-XL and CDK1/2/4 blockage we highlighted here point to this combination as a possible effective option to surpass, at least in part, the molecular heterogeneity of TNBC (e.g. the RB levels and its phosphorylation status). Future studies are needed to clarify whether BCL-XL $+\mathrm{CDK} 1 / 2 / 4$ inhibition would also minimize heterogeneous mechanisms of resistance otherwise occurring by targeting CDK4/6 with chemotherapeutics previously reported [40]. It would also be important to assess the net contribution of each CDK among the CDK1/2/4 inhibited by R547 while blocking BCL-XL. However, this would require rather laborious experimental setting using single and combined conditional shRNA sequences targeting each individual $\mathrm{CDK}$, as agents blocking specifically individual CDKs are not available. In relation to ongoing clinical trials using drugs blocking cell cycle regulators as targeted anticancer therapies, our studies underline the importance of carefully evaluating the best signals to target to achieve optimal response while minimizing side effects in patients.

Besides regulating cell cycle, new functions of CDK1/2/4 have recently emerged. It has been shown that CDK1/2 targets are hyperphosphorylated in basal-like breast cancer, generating genome integrity vulnerability [41]. Additionally, CDK1 phosphorylates several proteins involved in epigenetic regulation, such as the $\mathrm{H} 3 \mathrm{~K} 79$ methyltransferase Dot1l, responsible for placing activating marks on gene bodies [42]. Furthermore, it has been shown that CDK1 promotes storage of RTKs by suppressing endosomal degradation and recycling pathways [30].
The latter function may be particularly relevant for the effects we observed in TNBC cells following BCL-XL plus CDK1/2/4 targeting as most of the altered signals belong to RTK and AKT signalling, beside cell cycle regulators. In view of the findings reported in [30], it is tempting to speculate that inhibition of CDK1 perturbs RTK trafficking and storage, reducing the recycling pool due to increased processing and degradation. Lowering RTK and AKT signalling is likely detrimental for the cells, particularly in a context of reduced stress support pathway associated with BCL-XL inhibition. This also leads to nuclear retention, and therefore transcriptional activity, of FOXO3A, a tumour suppressor regulating expression of a plethora of genes involved in multiple biological processes. Thus, the effectiveness on TNBC cells of a combinatorial targeting of BCL-XL plus CDK1/2/4 inhibition likely resides on the acquisition of DNA damage (illustrated by an accumulation of $\gamma \mathrm{H} 2 \mathrm{AX}$ ) associated with cell cycle progression perturbation, an unbalance of survival/apoptotic signals (reduced XIAP-MCL1 and increased FASL-PUMA levels), with a concomitant depletion of RTK and AKT inputs. Future studies will define whether there is also a perturbation of epigenetic marks on gene bodies, as a consequence of CDK1/2 targeting [42]. This would be particularly relevant also in view of how high levels of oncogene sets are ensured by gene body hypermethylation, as we reported in liver cancer [17].

\section{Supplementary Material}

Supplementary figures and tables.

http://www.thno.org/v11p9180s1.pdf

\section{Acknowledgments}

We thank all members of our labs for helpful discussions and comments; R. Dono, F. Helmbacher, V. Géli for valuable feedback on the study; C. Sequera for help in processing data related to the overall survival of TNBC patients according to levels of cell cycle regulators; A.L. Bailly for performing cell cycle analysis reported in Figure 5D and Figure S3G; S. Richelme for assistance as lab manager; F. Daian for computational work to calculate the Synergy maps and Bliss scores; F. Argyriou for contributing to cell survival and western blot analyses; P. Perrin for providing us with mouse embryonic fibroblasts; and, the animal house platform for excellent help with mouse husbandry.

\section{Funding}

This research was supported by a grant from the Ministry of Foreign Affairs and International Development (MAEDI) and the Ministry of National 
Education, Higher Education and Research (MENESR) of France and by the Ministry of Science and Technology of Israel (Grant \#3-14002) to F.M. and S.L. This study was partly supported by research funding from Institut National du Cancer, Région Provence-Alpes-Côte d'Azur, and Canceropôle Provence-Alpes-Côte d'Azur to F.M. O.C. was supported by a fellowship from the Institut Cancer et Immunologie (Aix-Marseille Univ). F.A. was supported by the Higher Education Commission (HEC) of Pakistan. The contribution of the Région Provence-Alpes-Côte d'Azur and of the Aix-Marseille University to the IBDM animal facility and of the France-BioImaging/PICsL infrastructure (ANR-10INBS-04-01) to the imaging facility are also acknowledged. The funders had no role in study design, data collection and analysis, decision to publish or preparation of the manuscript.

\section{Author Contributions}

- O.C.: performed most of the biochemical experiments, cell viability assays, and computational work to analyse RNA-seq and RPPA outcomes; largely contributed to data analysis and interpretation.

- F.A.: performed the majority of the biochemical studies and cell viability assays, prepared samples for RPPA; data analysis and interpretation.

- Y.V.: performed computational work to analyse RNA-seq and RPPA outcomes, provided inputs on studies and on the manuscript.

- G.B.M.: contributed to RPPA studies; provided input on the manuscript.

- B.H.: processed RNA-seq outcomes and generated raw data; provided input on the manuscript.

- J.P.B.: supervised the FACS sorting analysis; provided input on the manuscript.

- S.L.: supervised RPPA analyses performed by Y.V.; provided input on studies and on the manuscript.

- F.L.: designed and supervised studies, contributed to experimental work, data analysis, and interpretation; contributed to write the manuscript.

- F.M.: designed and supervised the study, contributed to experimental work, analysed, and interpreted data, ensured financial support, and wrote the manuscript.

\section{Competing Interests}

The authors have declared that no competing interest exists.

\section{References}

1. Gayle SS, Sahni JM, Webb BM, Weber-Bonk KL, Shively MS, Spina R, et al. Targeting BCL-xL improves the efficacy of bromodomain and extra-terminal protein inhibitors in triple-negative breast cancer by eliciting the death of senescent cells. J Biol Chem. 2019; 294: 875-86.

2. Jhan JR, Andrechek ER. Triple-negative breast cancer and the potential for targeted therapy. Pharmacogenomics. 2017; 18: 1595-609.

3. Harbeck N, Penault-Llorca F, Cortes I, Gnant M, Houssami N, Poortmans P, et al. Breast cancer. Nat Rev Dis Primers. 2019; 5: 66.

4. Lamballe F, Ahmad F, Vinik Y, Castellanet O, Daian F, Muller AK, et al. Modeling Heterogeneity of Triple-Negative Breast Cancer Uncovers a Novel Combinatorial Treatment Overcoming Primary Drug Resistance. Adv Sci (Weinh). 2021; 8: 2003049

5. Garrido-Castro AC, Lin NU, Polyak K. Insights into Molecular Classifications of Triple-Negative Breast Cancer: Improving Patient Selection for Treatment. Cancer Discovery. 2019; 9: 176-98

6. Lev S. Targeted therapy and drug resistance in triple-negative breast cancer: the EGFR axis. Biochem Soc Trans. 2020; 48: 657-65.

7. Zhang S, Shao Y, Hou G, Bai J, Yuan W, Hu L, et al. QM-FISH analysis of the genes involved in the G1/S checkpoint signaling pathway in triple-negative breast cancer. Tumour Biol. 2014; 35: 1847-54.

8. Rocca A, Schirone A, Maltoni R, Bravaccini S, Cecconetto L, Farolfi A, et al. Progress with palbociclib in breast cancer: latest evidence and clinical considerations. Ther Adv Med Oncol. 2017; 9: 83-105.

9. Balko JM, Giltnane JM, Wang K, Schwarz LJ, Young CD, Cook RS, et al. Molecular profiling of the residual disease of triple-negative breast cancers after neoadjuvant chemotherapy identifies actionable therapeutic targets. Cancer Discov. 2014; 4: 232-45.

10. Otto T, Sicinski P. Cell cycle proteins as promising targets in cancer therapy. Nat Rev Cancer. 2017; 17: 93-115.

11. Rubin SM, Sage J, Skotheim JM. Integrating Old and New Paradigms of G1/S Control. Molecular Cell. 2020; 80: 183-92.

12. Liu CY, Lau KY, Hsu CC, Chen JL, Lee CH, Huang TT, et al. Combination of palbociclib with enzalutamide shows in vitro activity in RB proficient and androgen receptor positive triple negative breast cancer cells. Plos One. 2017; 12: e0189007.

13. Yamamoto T, Kanaya N, Somlo G, Chen SA. Synergistic anti-cancer activity of CDK4/6 inhibitor palbociclib and dual mTOR kinase inhibitor MLN0128 in pRb-expressing ER-negative breast cancer. Breast Cancer Res Tr. 2019; 174: $615-25$.

14. Cretella D, Fumarola C, Bonelli M, Alfieri R, La Monica S, Digiacomo G, et al. Pre-treatment with the CDK4/6 inhibitor palbociclib improves the efficacy of paclitaxel in TNBC cells. Sci Rep. 2019; 9: 13014.

15. Ge JY, Shu S, Kwon M, Jovanovic B, Murphy K, Gulvady A, et al. Acquired resistance to combined $\mathrm{BET}$ and $\mathrm{CDK} 4 / 6$ inhibition in triple-negative breast cancer. Nat Commun. 2020; 11: 2350.

16. Ianevski A, He L, Aittokallio T, Tang J. SynergyFinder: a web application for analyzing drug combination dose-response matrix data. Bioinformatics. 2017; 33: $2413-5$

17. Arechederra M, Daian F, Yim A, Bazai SK, Richelme S, Dono R, et al. Hypermethylation of gene body $\mathrm{CpG}$ islands predicts high dosage of functional oncogenes in liver cancer. Nat Commun. 2018; 9: 3164.

18. Dobin A, Davis CA, Schlesinger F, Drenkow J, Zaleski C, Jha S, et al. STAR: ultrafast universal RNA-seq aligner. Bioinformatics. 2013; 29: 15-21.

19. Liao Y, Smyth GK, Shi W. featureCounts: an efficient general purpose program for assigning sequence reads to genomic features. Bioinformatics. 2014; 30: 923-30.

20. Love MI, Huber W, Anders S. Moderated estimation of fold change and dispersion for RNA-seq data with DESeq2. Genome Biol. 2014; 15: 550.

21. Subramanian A, Tamayo P, Mootha VK, Mukherjee S, Ebert BL, Gillette MA, et al. Gene set enrichment analysis: a knowledge-based approach for interpreting genome-wide expression profiles. Proc Natl Acad Sci U S A. 2005; 102: 15545-50

22. Mootha VK, Lindgren CM, Eriksson KF, Subramanian A, Sihag S, Lehar J, et al. PGC-1alpha-responsive genes involved in oxidative phosphorylation are coordinately downregulated in human diabetes. Nat Genet. 2003; 34: 267-73.

23. Hong Z, Wang Q, Hong C, Liu M, Qiu P, Lin R, et al. Identification of Seven Cell Cycle-Related Genes with Unfavorable Prognosis and Construction of their TF-miRNA-mRNA regulatory network in Breast Cancer. J Cancer. 2021; 12. $740-53$

24. DePinto W, Chu XJ, Yin XF, Smith M, Packman K, Goelzer P, et al. In vitro and in vivo activity of R547: a potent and selective cyclin-dependent kinase inhibitor currently in phase I clinical trials. Mol Cancer Ther. 2006; 5: 2644-58.

25. Giacinti C, Giordano A. RB and cell cycle progression. Oncogene. 2006; 25: 5220-7.

26. Koo CY, Muir KW, Lam EWF. FOXM1: From cancer initiation to progression and treatment. Bba-Gene Regul Mech. 2012; 1819: 28-37. 
27. Lahne HU, Kloster MM, Lefdal S, Blomhoff HK, Naderi S. Degradation of cyclin D3 independent of Thr-283 phosphorylation. Oncogene. 2006; 25: 2468-76.

28. Greider C, Chattopadhyay A, Parkhurst C, Yang E. BCL-x(L) and BCL2 delay Myc-induced cell cycle entry through elevation of p27 and inhibition of G1 cyclin-dependent kinases. Oncogene. 2002; 21: 7765-75.

29. Schmitt E, Beauchemin M, Bertrand R. Nuclear colocalization and interaction between bcl-xL and $\operatorname{cdk} 1(\mathrm{cdc} 2)$ duringG(2)/M cell-cycle checkpoint. Oncogene. 2007; 26: 5851-65.

30. Cota CD, Dreier MS, Colgan W, Cha A, Sia T, Davidson B. Cyclin-dependent Kinase 1 and Aurora Kinase choreograph mitotic storage and redistribution of a growth factor receptor. PLoS Biol. 2021; 19: e3001029.

31. Liu Y, Ao X, Ding W, Ponnusamy M, Wu W, Hao X, et al. Critical role of FOXO3a in carcinogenesis. Mol Cancer. 2018; 17: 104

32. Koboldt DC, Fulton RS, McLellan MD, Schmidt H, Kalicki-Veizer J, McMichael JF, et al. Comprehensive molecular portraits of human breast tumours. Nature. 2012; 490: 61-70.

33. Solimini NL, Luo J, Elledge SJ. Non-oncogene addiction and the stress phenotype of cancer cells. Cell. 2007; 130: 986-8.

34. Raychaudhuri P, Park HJ. FoxM1: A Master Regulator of Tumor Metastasis. Cancer Res. 2011; 71: 4329-33.

35. Tan YL, Wang QX, Xie YB, Qiao XX, Zhang S, Wang YA, et al. Identification of FOXM1 as a specific marker for triple-negative breast cancer. Int J Oncol. 2019; 54: 87-97.

36. Dey P, Wang A, Ziegler Y, Kim SH, El-Ashry D, Katzenellenbogen JA, et al. Suppression of Tumor Growth, Metastasis, and Signaling Pathways by Reducing FOXM1 Activity in Triple Negative Breast Cancer. Cancers (Basel). 2020; 12 .

37. Ring A, Nguyen C, Smbatyan G, Tripathy D, Yu M, Press M, et al. CBP/beta-Catenin/FOXM1 Is a Novel Therapeutic Target in Triple Negative Breast Cancer. Cancers (Basel). 2018; 10

38. Huang $\mathrm{Y}, \mathrm{Wu} \mathrm{H}, \mathrm{Li}$ X. Novel sequential treatment with palbociclib enhances the effect of cisplatin in RB-proficient triple-negative breast cancer. Cancer Cell Int. 2020; 20: 501.

39. Yang N, Wang C, Wang J, Wang ZF, Huang D, Yan M, et al. Aurora kinase A stabilizes FOXM1 to enhance paclitaxel resistance in triple-negative breast cancer. J Cell Mol Med. 2019; 23: 6442-53.

40. Asghar US, Barr AR, Cutts R, Beaney M, Babina I, Sampath D, et al. Single-Cell Dynamics Determines Response to CDK4/6 Inhibition in Triple-Negative Breast Cancer. Clin Cancer Res. 2017; 23: 5561-72.

41. Sviderskiy VO, Blumenberg L, Gorodetsky E, Karakousi TR, Hirsh N, Alvarez SW, et al. Hyperactive CDK2 Activity in Basal-like Breast Cancer Imposes a Genome Integrity Liability that Can Be Exploited by Targeting DNA Polymerase epsilon. Mol Cell. 2020; 80: 682-98 e7.

42. Michowski W, Chick JM, Chu C, Kolodziejczyk A, Wang Y, Suski JM, et al. Cdk1 Controls Global Epigenetic Landscape in Embryonic Stem Cells. Mol Cell. 2020; 78: 459-76 e13. 


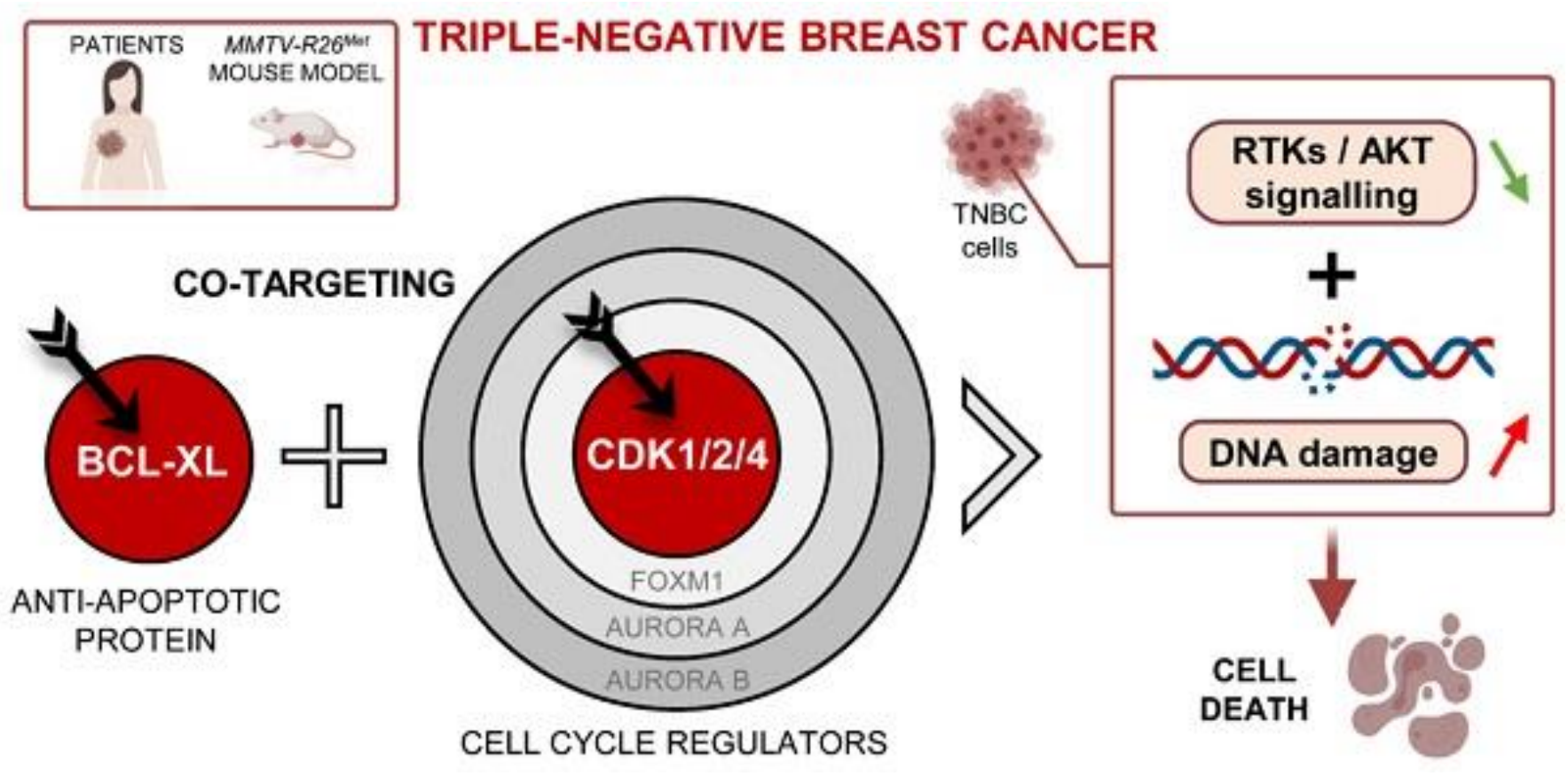

